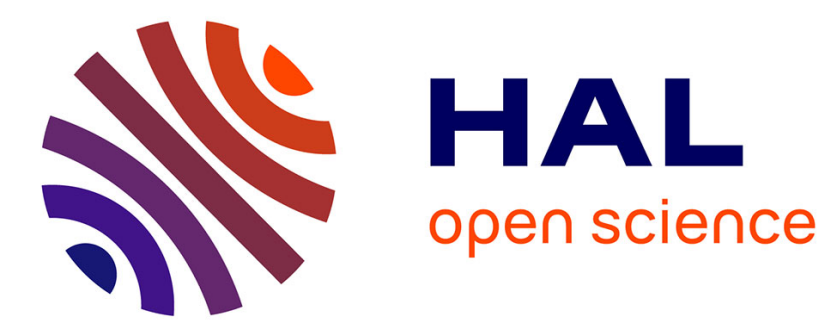

\title{
Compartmental Modelling in chemical engineering: A critical review
}

Nicolas Jourdan, Thibaut Neveux, Olivier Potier, Mohamed Kanniche, Jim Wicks, Ingmar Nopens, Usman Rehman, Yann Le Moullec

\section{To cite this version:}

Nicolas Jourdan, Thibaut Neveux, Olivier Potier, Mohamed Kanniche, Jim Wicks, et al.. Compartmental Modelling in chemical engineering: A critical review. Chemical Engineering Science, 2019, 210, pp.115196 -. 10.1016/j.ces.2019.115196 . hal-03487346

\section{HAL Id: hal-03487346 \\ https://hal.science/hal-03487346}

Submitted on 21 Dec 2021

HAL is a multi-disciplinary open access archive for the deposit and dissemination of scientific research documents, whether they are published or not. The documents may come from teaching and research institutions in France or abroad, or from public or private research centers.
L'archive ouverte pluridisciplinaire HAL, est destinée au dépôt et à la diffusion de documents scientifiques de niveau recherche, publiés ou non, émanant des établissements d'enseignement et de recherche français ou étrangers, des laboratoires publics ou privés.

\section{다)(1) $(5$}

Distributed under a Creative Commons Attribution - NonCommerciall 4.0 International 


\title{
Compartmental Modelling in Chemical Engineering : A Critical Review
}

\author{
Nicolas Jourdan ${ }^{\mathrm{a}, \mathrm{b}}$, Thibaut Neveux ${ }^{\mathrm{b}}$, Olivier Potier ${ }^{\mathrm{a}, *}$, Mohamed Kanniche $^{\mathrm{b}}$, Jim Wicks ${ }^{\mathrm{c}}$, Ingmar Nopens ${ }^{\mathrm{d}}$, \\ Usman Rehman ${ }^{\mathrm{e}}$, Yann Le Moullec ${ }^{\mathrm{f}}$ \\ ${ }^{a}$ Laboratoire Réactions et Génie des Procédés (LRGP), UMR 7274 CNRS, Université de Lorraine, Nancy F-54001, France \\ ${ }^{b}$ EDF R\&D, EDF Lab Chatou, 6 quai Watier, 78401 Chatou Cedex, France \\ ${ }^{c}$ The Fluid Group, The Magdalen Centre, Robert Robinson Avenue, The Oxford Science Park, Oxford OX4 4GA, UK

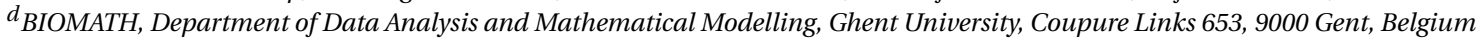

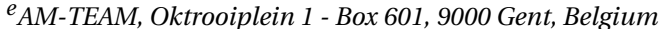

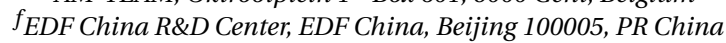

\begin{abstract}
Compartmental modelling is a hybrid way to model complex systems in Chemical Engineering. This modelling approach offers numerous advantages because it aggregates information from both local and system scale models. Compartmental models allow multi-scale modelling with low computational time compared to a full coupled model (e.g. reactive numerical simulations). Thanks to these main characteristics, compartmental models are able to model complex full-size industrial systems.

For the last decades, various approaches of compartmental models were developed for different applications. In this article, a critical review and analyses are carried out to classify these different approaches. A unified definition is proposed, and important guidelines are pointed out to assist with constructing a Compartmental Model.

Finally, some perspectives for the future of Compartmental Modelling are identified and discussed: compartmental modelling for larger and more complex systems, the inclusion of new phenomena modelling and automation of compartmental models with the improvement of numerical methods.
\end{abstract}

Keywords: Compartmental Modelling, Hydrodynamics, Multi-scale, CFD, Systemic Model

\section{Introduction}

In chemical engineering the modelling of all the relevant phenomena, at appropriate scale, in industrial systems is a scientific challenge. Developing accurate models of multi-phenomenon systems, which typically exhibit nonlinear behaviour, requires an important amount of data and faces the capacity limits of available technologies and measurement techniques. Different modelling approaches are used depending on the purpose of the study and the objectives of the constructed model. The hybrid method called compartmental approach is gaining popularity. For twenty years, the number of industrial applications using compartmental model approaches continues to increase (e.g. bioreactors [84], water treatment [91], crystallization [80], $\mathrm{CO}_{2}$ valorisation [116], gas turbine modelling [31]...). The purpose of this work is to make an inventory of the different compartmental modelling approaches developed in the literature so far and to analyze the different models in order to propose a definition for compartmental modelling in chemical engineering.

Depending on the modelling objectives, models should be able to describe, explain or predict a system behaviour, it generally consists of a set of equations representing the key aspect of the system of interest, and the relevant boundary conditions, aiming to take into account all the important phenomena occurring in the system and their dependencies (transfer, transport, reactions, phase transition) at local or global scale (Potier et al. [87]), A model can never be complete but must offer a good balance between complexity and accuracy therefore relevant

\footnotetext{
* Corresponding author

Email address: olivier .potier@univ-lorraine.fr (Olivier Potier)
} 
simplification is almost always needed. Designing a model will be guided by the specific system studied and scale, the study objectives, the available information and theoretical knowledge as well as the capability to process this information (i.e. the need or not of special competencies, powerful computer, etc.).

In systems, within chemical engineering field of study, one the most important phenomena is the system dynamics, which often induces the overall model structure. Most phenomena studied are partially, or fully, linked to fluid dynamics behaviour of the system as a consequence, numerous studies focus on fluid dynamics models of unit operation (Dudukovic [29]). Developing a model could be a very complex operation, two approaches are commonly used to model fluid dynamics industrial systems: the systems approach and the local approach that refers to a Cartesian approach in epistemology (de Rosnay [24]).

Modelling approaches use information from studies at system (global) scale or local scale or both. The system scale considers the whole system as an undivided fashion. The system characterisation is done by using inlet and outlet data (tracing, Residence Time Distribution...), calculating global quantities (e.g. global transfer performance, residence time, conversion, mass balance, etc.). In contrast, the local scale tries to characterize occurring phenomena; it is commonly set in opposition to the system scale. The local scale allows the calculation of quantities such as species concentration, fluid velocity and turbulence fields, local transfer constants, etc., distributed within the studied system. Computational Fluid Dynamics (CFD) uses the local approach to model the whole system, a reactor for example. That is why, the local scale can be different depending on occurring phenomena. In the following chemical engineering studies, the local scale is often set as the fluid dynamics resolution in the system study.

This work aims to present the history of Compartmental Modelling approach development through the critical review of compartmental models developed in the literature during the past decades. Based on this review, a critical analysis of the diversity of compartmental approaches is done focusing on construction specificity. Thanks to the analysis of literature, a unified definition and a general construction methodology are developed and proposed. Finally, some considerations and perspectives for compartmental modelling futures are discussed and shared.

\section{Process modelling in chemical engineering}

In this part, the different modelling approaches in chemical engineering are introduced and described. The systemic and local approaches are explained to point out the necessity to develop a new complementary modelling approach.

\subsection{Systemic approach}

The systemic approach originally considered the system as a black box, in which progressively information and models are added as the tools and the needs progress. In our domain, the system is the whole reactor, column or volume of interest. This fruitful approach came out in chemical engineering from the use of hydrodynamic analysis method: tracing that is based on information from input and output (Danckwerts [21]). Rapidly, successful methods were proposed to model the global fluid dynamics of a complex reactor with combinations of a few "virtual" ideal reactors: Continuous Stirred-Tank Reactors (CSTRs) and Plug Flow Reactors. In their respective reference works concerning modelling in chemical engineering, Villermaux [103, 104] and Levenspiel [64] explain and illustrate this approach. The determination of the overall hydrodynamics is done by the study of input and output data resulting in the calculation of system conversion and global balances. Obviously, the main tool for reactor networks determination is fluid tracing. A known tracer distribution is injected in input and measurements of the output give the Residence Time Distribution (RTD) functions as explained in Danckwerts [21]. The analysis of RTD functions gives a possible network of "virtual" ideal reactors that is representative of the global fluid dynamics system (see Figure 1 left) (Hocine et al. [37], Laquerbe et al. [53]). The representative structure is not necessarily unique and could be improved by getting information such as geometry (reactor shape, size) or mixing device position and energy provided to the fluid. Once the network of ideal reactor is determined, other phenomena can be added in the network. The resulting model is representative of the influence of hydrodynamics on the other phenomena ; this representation is rigorously true for linear phenomena only but experience shows that appropriate representation of almost all system can be appropriately described by this approach. It has been 


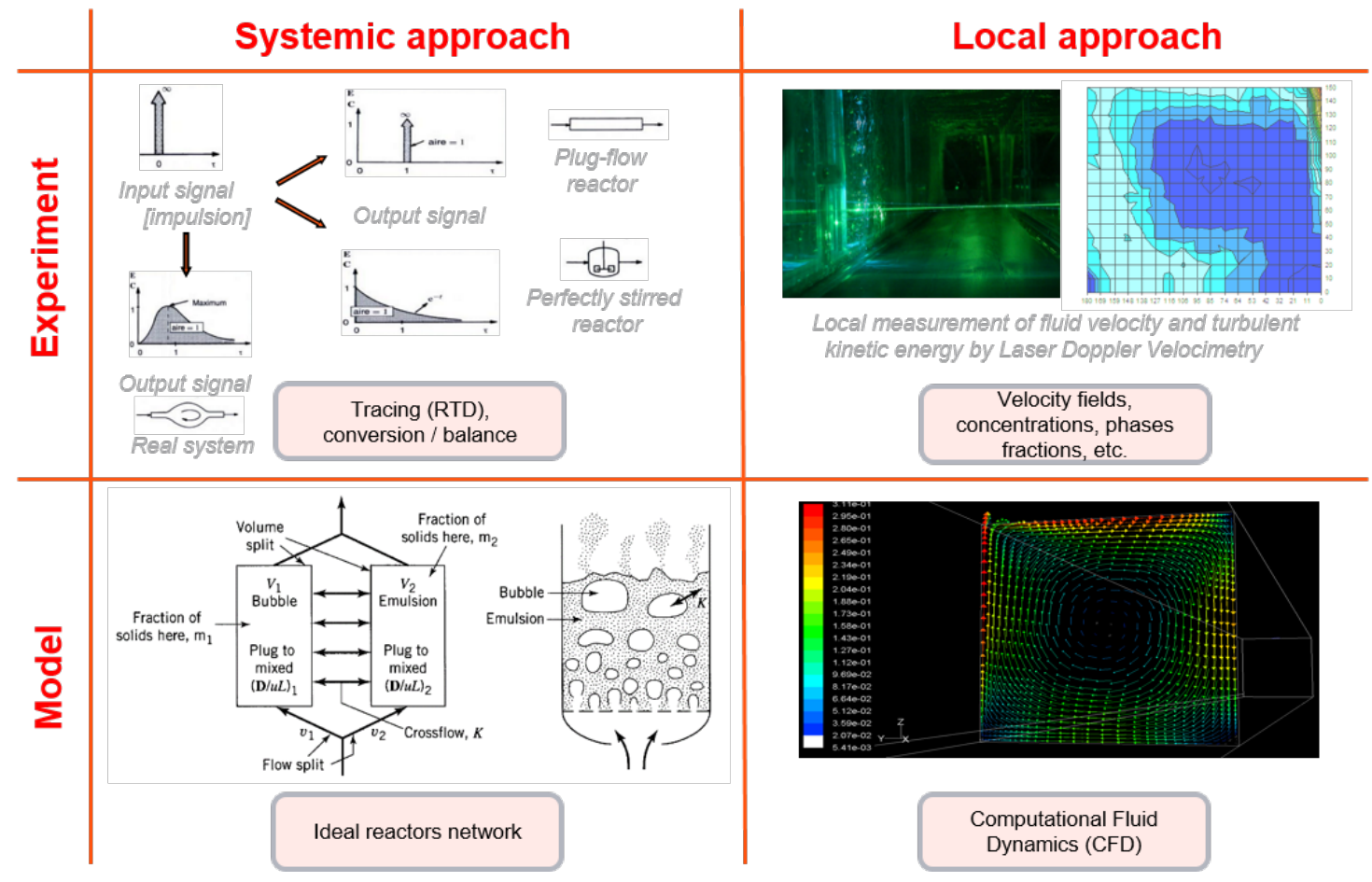

Figure 1: Comparison between systemic and local approaches (images adapted from Levenspiel [64] and Le Moullec [58])

commonly used in all fields of chemical engineering such as: water treatment (Laurent et al. [57], Potier et al. [86], Stropky et al. [98]), combustion (Faravelli et al. [30]), polymerisation (Vivaldo-Lima et al. [106]), multi-stage contactors (Zhang et al. [115]) or multiphase flow systems (Simcik et al. [97]). The systemic approach with reactors networks are generally used in process modelling, process development, and scale-up, in order to scan all the combination scenarios and rapidly obtain a first design with sufficient precise results of a new process or before an improvement of an existing one. At the beginning, the systemic approach was often called "compartment model or modelling" (Levenspiel [64]). Progressively, the comparison with other sciences such as cybernetics, general system theory, automation, epistemology (von Bertalanffy [107], de Rosnay [24], Villermaux [103, 104], Morin [73]) induced a change in the terminology ensuring consistency with them. It is important to note that this older naming (compartment modelling) is quite different from the compartmental modelling that is the subject of this article. In the post WW2 era, the emergence of the systemic approach helped the fruitful development of Chemical Reaction Engineering (CRE) as a new discipline (Dudukovic [29]). Afterwards, they evolved concomitantly. Using mainly the systemic approach during decades, CRE helped with high efficiency to design and optimize processes. CRE mainly combines kinetics, thermodynamics, transfer and hydrodynamics that are modelled with the systemic approach. However, CRE evolved with time and now it also uses other approaches such as local approach, CFD with reactions for example.

\subsection{Local approach}

Complementary to system scale information, it could be important to also get local information. At local scale, two main methods are used to characterize the fluid dynamics behaviour: local experimental measurements or numerical simulations (see Figure 1 right). The two approaches can be complementary, they can be used both at the same time or successively; for instance, local measurements give information for the model building and are also used to validate the model. A significant number of data acquisition methods can be used depending on the flow pattern such as interface characteristics (Takagaki and Komori [100]), disperse and continuous phase characteristics (Lee [63], Tibirica et al. [101]), chemical data (Dani et al. [22]), fluid transport properties and ther- 
mohydraulics properties (Goldstein [33]). Another way to get some data on fluid dynamics is local simulation of fluid dynamics fields by solving the equations of fluid mechanics (Versteeg and Malalasekera [102]).

CFD development is benefiting for many years from continuous increase in computer capacity and applications. They can therefore provide more and more accurate data on more and more complex flows and geometries. The simulation accuracy depends on the system discretisation (or meshing) and on the approach taken for solving Navier-Stokes equations and other scalar variables: solving these equations can provide more accurate simulations of the main fluid dynamics quantities of interest such as velocity, pressure, temperature, passive scalars, such as species concentration, or turbulence. The most common CFD approaches are not an exact representation of the system because they rely on a chosen set of hypothesis and on turbulence models for some approaches. For multiphase flows, different simulation methods can be used such as two-fluids Euler-Euler, Volume of Fluid (VOF), level set or Lattice Boltzmann...

For most of complex flow occurring in complex geometry, only a statistical average form of CFD equations can be solved, which provides mean values of variables. With this approach, the geometry does not need a very fine discretisation, which enables to reduce the calculation capacity demand. When averaging Navier-Stokes equations, non-linear terms appear as mean turbulence correlations that are solved using turbulence modelling: linear eddy viscosity models (Chen and Xu [19], Menter [71], Scott-Pomerantz [95]), non-linear eddy viscosity models (Laurence et al. [56], Wizman et al. [112]) and Reynolds Stress Models ([55, 112]). Non-statistical approaches such as Direct Numerical Simulation (DNS) directly solve Navier-Stokes equations without any statistical operation nor turbulence model (Moin and Mahesh [72]). Much finer meshes are therefore needed in order to catch the energy dissipation at smallest scales (Kolmogorov micro-scales). This exact DNS approach is still too time consuming to be applied to large and complex flows occurring in complex geometries. To reduce computational cost of DNS the Large Eddy Simulation (LES) method was developed in the seventies. The small scales of Navier-Stokes equations are ignored to only solved larger scales. . The LES approach is nowadays applied to very complex flows occurring in complex geometries such as turbulent combustion (Pitsch [85]) but is still time consuming and difficult to apply to multi-phase flows.

CFD simulations can be a useful tool for many flows simulations in many scientific domains: examples of the use of CFD simulations in various domains are listed in Angermann [6].

\subsection{Limitations of traditional approaches and compartmental modelling approach}

The two previous modelling approaches have both advantages and drawbacks. They are highly complementary for system studies or reactors design.

Systemic approaches are studied for decades and rely on ideal reactors whose fluid dynamics equations are easy to solve. These equations only take into account a few global parameters (e.g. inlet and outlet flow rates, reactor volume, residence time, number of "virtual" ideal reactor). The solution difficulty can increase with the complexity of ideal reactor network, but a global fluid dynamics model can always be obtained. Moreover, the systemic approach usually does not depend on the system size and can be used for both lab scale or industrial scale systems with the same procedure and calculation time. However, systemic approaches only give global information and are often insufficient to fully understand a system or a phenomenon. Due to the multiplicity of RTD functions and the various possible reactor networks, systemic models are not representative in detail of the actual geometry of the system because occuring phenomena are not spatially defined.

Local measurements and simulations of fluid dynamics provide data for various parameters of the flow pattern (e.g. local velocities, viscosity, phase fractions, temperature, etc.). These local data characterize the system geometry, operating conditions and provide the actual mapping of the system. For complex systems and geometries, local approaches could remain costly: to obtain a complete experimental characterisation, an important experimental setup is needed to get enough experimental values for a consistent mapping. Moreover, invasive probes can perturb the fluid dynamics behaviour of the system. Local characterisation and particularly CFD simulations remain complicated to perform and sometimes, just unfeasible. If all the coupled phenomena are taken into account in complex systems (e.g. complex reactive systems, quick combustion reactions, strongly coupled phenomena), the number of solved equations explodes. The increase of calculation capacity will certainly improve the scope of CFD simulation but the gap remains huge enough to reach the full modelling of an industrial scale complex system. For now, some physical phenomena are impossible to fully comprehend at local scale such 
as particle deposition or heterogeneous systems (when a same system combines different unit operation within a same location, it often happens in process intensification). To overcome the historical modelling approaches limitations, the compartmental approach was developed in the last twenty years to combine the advantages of both local and systemic approaches.

A lot of compartmental models were developed for various study cases using different construction methods but the same philosophy of construction remains. Previous works already pointed out the particularities of compartmental modelling in comparison with systemic modelling (Haag et al. [36]). This work addresses a detailed analysis of compartmental model evolution and proposes a first specific definition of this approach.

The next section reviews compartmental modelling approaches described in the literature; afterward, the justification of compartmental approaches use is explained.

\section{Review of compartmental approaches}

All the compartmental approaches have a common theory of model construction, but also significant differences in the choice of compartment definition criteria. The construction methods have been adapted to the studied systems. The main compartmental approaches developed in the literature are listed and detailed in Table 1, focusing on industrial applications, phenomena of interest and goals of the model development.

The goal of section 3. is to present a description of compartmental models as complete as possible.

\subsection{Empirical approach for compartment construction}

The origins of compartmental models go back to the 70's [81], but until the middle of the nineties, the name "compartment models" was used to describe a network of ideal reactors. In most cases, compartment models referred to systemic models (Cui et al. [20], Mayr et al. [70], Reuss and Jenne [92]). Vrabel et al. [108, 109, 110] used the same empirical construction method to model a multi impeller stirred tank. The compartment definition was empirically based on reactor geometry and local hydrodynamics knowledge from previous studies: each impeller zone is divided in a network of ideal reactors. This study points out the importance of exchange fluxes calculation that will be a key aspect in designing compartment networks.

The first compartmental approaches were developed in the late nineties to get some complete models of reactors in spite of the low capacity of CFD simulations. Despite the use of CFD in the model construction process, these models were mainly empirically based: the compartments were designed with available knowledge about studied reactor hydrodynamics. To control the process of Czochraslski crystallization, Irizarry-Rivera and Seider [41, 42] and Irizarry-Rivera [40]developed a first type of compartmental model. The objective was to model the convection in the crystallizers and its influence on crystal growth. Local information about hydrodynamics came from the available models such as the boundary layer model and empirical flow field predictions. Then, the bulk part of the reactor was divided in a Continuous Stirred Tank Reactor network.

In 1999, Bauer and Eigenberger [9, 10] developed a 'multi-scale zone model', precursor of the idea of compartmental modelling. The multi-scale approach gathers information from simulated hydrodynamics, mass transfer models and global balances. The detailed fluid dynamics allows the division of the reactor in 'zones' to get a simplified hydrodynamic model. This simplified model was very simple and the reactor network was not complex. Population balance, mass transfer and reaction models were added on this simplified reactor model to get the complete bubble column model. The Bauer model was an iterative process with the defined compartment structure: CFD simulations provides exchange fluxes and the multi-zonal model provide the two phases interaction characteristics as an input for the CFD simulations (2).

Using the same construction scheme to study mixing quality and turbulence in stirred tanks, Alexopoulos et al. [3], Maggioris et al. [68,69] developed a simple two-compartment model based on the reactor geometry: one compartment for the impeller zone and the other representing the remaining volume. A complete CFD simulation of flow fields and turbulence were not used in the compartment definition but its results has been added to the empirical two-compartment model to enhance the reactor description. Each compartment is independent and includes its own turbulence parameters from CFD. Bashiri et al. [7, 8] used the same empirical division of a stirred tank in two compartments to study the sensibility of the model for reactor scale-up. The boundaries of the two compartments were determined thanks to the investigation of turbulent energy dissipation which is 
representative of the mixing: an empirical value of energy dissipation rate is set as the cut-off value for the boundary determination. The compartmental model was enhanced with different CFD simulation results from three geometrical similar reactors with different volumes from 7 to 465 litres.

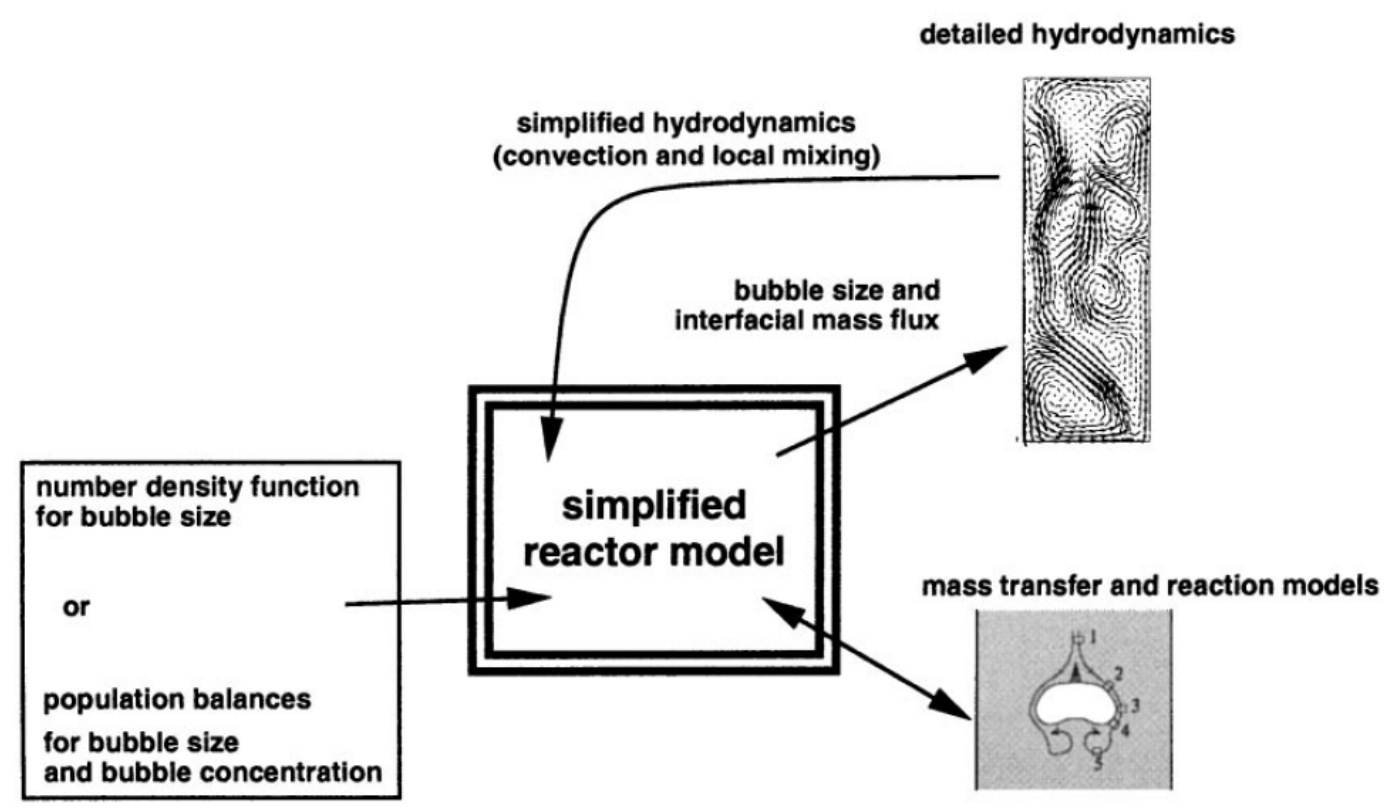

Figure 2: Multi-scale model of Bauer and Eigenberger [9]

\subsection{Compartments construction based on fields calculated by CFD}

With the improvement of computer simulation power, the first CFD based compartmental models were established. Bermingham et al. [13, 14] developed a compartmental model to design a crystallizer. They identified the parameters that influences the crystallization mechanisms (supersaturation, energy dissipation and crystal size) and ran CFD simulation of hydrodynamics. They selected the compartments using the flow pattern and the energy dissipation field of simulations results. The created compartments were then checked for the homogeneity of the two other criteria conditions: supersaturation gradient and crystal size distribution.

Kougoulos et al. [48] use the same construction method as Bermingham and coworkers for the same industrial system to study mixing and heat transfer. They completed the Bermingham approach including the temperature as compartment definition criteria. The compartmental model remains based on simplified hydrodynamic simulations. A complete model of particle conservation and crystallization kinetics is implemented in the compartments considered as perfectly Stirred Tanks.

To study aerobic xanthan fermentation in a stirred tank, Laakkonen et al. [50, 49, 51] also used an empirical way to define compartments: relative parameters homogeneity within a compartment and constant parameter gradient along compartment frontiers. Empirically, Laakonnen also minimise the number of compartments within a flow direction to obtain a suitable model for different xanthan concentrations.

Alex et al. [1,2] built a CFD based compartmental model considering a 'decomposition property' simulated by CFD. This criterion allows the division of the reactor into compartments and exchanges flows are calculated by mass balance. In this example of a water treatment canal reactor, the decomposition criterion is the homogeneity of fluid particles age within a compartment. Finally, each compartment is considered as a Continuous Stirred Tank Reactor .

The objective of Bezzo et al. [18], Bezzo and Macchietto [15] was to model bioreactors. It was the first study developing a cell aggregation method. The first step is to construct a simple multi-zonal model corresponding to a spatial division of the reactor into compartments: the division is based on the homogeneity of global parameters. Then, the hybrid multizonal/CFD model is built adding the results of CFD simulations to get the non- 
homogeneous flow parameters. The two models are coupled with an iterative process: the simulation results provide flow parameters to aggregate the cells corresponding to an empirical zone and the multi-zonal model provides physical properties parameters. The next study of Bezzo and Macchietto [16] explains how to optimise the compartment definition with a practical algorithm and how to reduce the final number of zones to simplify the model.

The Delafosse et al. [25, 26] method is based on the Bezzo and coworkers approach. The modelling of a two impeller bioreactor is done by using a first spatial compartment definition. The chosen number of compartments is important to accurately model the recirculation loops that impact the studied mixing time. This study aimed to improve the compartment construction from CFD results comparing several cell aggregation methods: the cellby-cell method also used by Bezzo and the layer-by-layer method. This study also pointed out the importance of mean and turbulent flowrates between the compartments. Oner et al. [80] and Norregaard et al. [78] used the same compartment construction method to study scale-up modelling for pharmaceutical crystallization process and analyse mixing in bioreactors.

Pigou and Morchain [84] developed a model to represent biological population balance. The hydrodynamic behaviour of the reactor is based on the Vrabel et al. $[108,109,110]$ study but the constructed compartmental model was then completed with a population balance model for the population dynamics and a metabolic model for bioreactions representation.

A compartmental population balance model was developed by Yu et al. [114] for a High Shear Granulation process. The heuristic construction of the compartmental model is based on the previous approaches for crystallizers. The compartment definition is done analyzing flow patterns, solid particle velocity and solid concentrations obtained by CFD simulations. Each compartment is considered as a Continuous Stirred Tank Reactor and the model is used to run a distributed macroscopic model.

\subsection{Rationalisation of compartments construction}

After introducing newly (at this time) CFD results in compartmental model design, the next key step in Compartmental Models development has been the rationalisation of compartments construction. The number of compartments is no longer chosen by the study author (based on previous similar studies or chosen after an iterative process of model construction), but it relies on set values of the construction criteria within the definition algorithm. The rationalisation of compartments construction allows a better adaptability of the built model, but is more complex to implement.

The Rigopoulos and Jones [93] construction method follows the same scheme as the previous ones. The compartments are established in order to respect hydrodynamics conditions. A simplified CFD simulation was run and the compartments were defined following two criteria: the flow must be unidirectional along the compartments frontiers and the flow age must be homogeneous within a compartment. Then, each compartment with important dispersed phase fractions must be divided in two interconnected sub-compartments: one sub-compartment for highly dispersed phase fractions and one for the low fraction. This approach is the first one focusing on the exchange fluxes between compartments. The convective exchanges are calculated with mass balance between compartments and transfer phenomena are represented by exchange fluxes between the sub-compartments. To validate the model, a numerical RTD is simulated with the compartmental model and compared to experimental data. If the results do not match, the precision of the compartment definition must be improved.

The Guha et al. [35] approach is close to the Rigopoulos and coworkers approach: simplified CFD simulations are used as bases for the compartments determination with more accurate definition criterion. The compartment definition is done investigating the velocity fields and studying the respective phenomena time scales. If the reaction time scale is significantly higher than the convection time scale, then hydrodynamic behaviour can be considered as independent from reactions. The residence time of the liquid in a compartment must be shorter than the characteristic reaction time scale in order to avoid the creation of a concentration gradient due to chemical reactions. The convective fluxes are calculated solving mass balance equations and the turbulent fluxes are calculated using the results of turbulence fields simulations.

Wells and Ray [111] used a compartmental approach to study mixing effects in complex polymerisation reactors. They first ran a simplified CFD simulation with the reaction of the autoclave reactor; they wanted a model to obtain temperature and concentration fields. The reactive simulation was run with 100,000 cells and with a 
strong rotational hypothesis concerning turbulent flow quantities to save computational time. The compartments were built splitting successively the initial reactor into compartments until a preset number of compartments is obtained. The compartments are divided to reduce the internal variation of temperature and the discrepancy between a compartment volume summed kinetic rate and the rate that would occur on average conditions. The reaction rates are calculated thanks to the simulated temperature and concentration fields. The construction method is applied to obtain a 100 compartment model for a stirred autoclave reactor of low-density polyethylene.

Le Moullec et al. $[61,62]$ used the same general scheme of compartmental model construction using CFD simulations and system partition criteria. These criteria are not based on exchange fluxes like the Rigopoulos method nor on time scales like the Guha and coworkers approach but they are based on the system physical-chemical properties such as gas fractions, turbulence dissipation (mixing intensity) and velocity fields. The empirical analysis of these properties induce the radial partition of the canal reactor in compartments. Then, the axial partition is done using results of global RTD experiments and simulations. Exchanged convective fluxes are calculated integrating simulated velocity fields and turbulent fluxes are determined analysing compartments by pairs. Finally, the Activated Sludge Model 1 model for the chemical and biological reactions of water treatment was added to the model to obtain the functional compartmental model (Figure 3).

Iliuta et al. [38, 39] developed a compartmental approach to model bubble columns. Because of the difficulty of multi-phases and multi-component problems, Iliuta used a local hydrodynamic model developed for slurry bubble column to identify the compartments. A mathematical condition is set to the obtained velocity fields and bubble distribution profiles: homogeneity of fluid velocity and gas fraction within a compartment. The compartmental model is completed with thermodynamic and kinetic models linked to the Fischer-Tropsh process.

Gresch et al. [34] focuses on the turbulent exchanges in the compartmental approaches with an application to wastewater canal reactors. A first simulation of steady state hydrodynamics is run with a transport model of reactive species. The CFD cells are aggregated to obtain compartments following iso-concentration zones and homogeneous flow directions. Then, convective fluxes are calculated integrating velocity fields at the compartments frontiers. Gresch considers that the compartment model already includes inner turbulence. Then turbulent fluxes are calculated with CFD turbulent fields with a turbulence coefficient adaptation. 


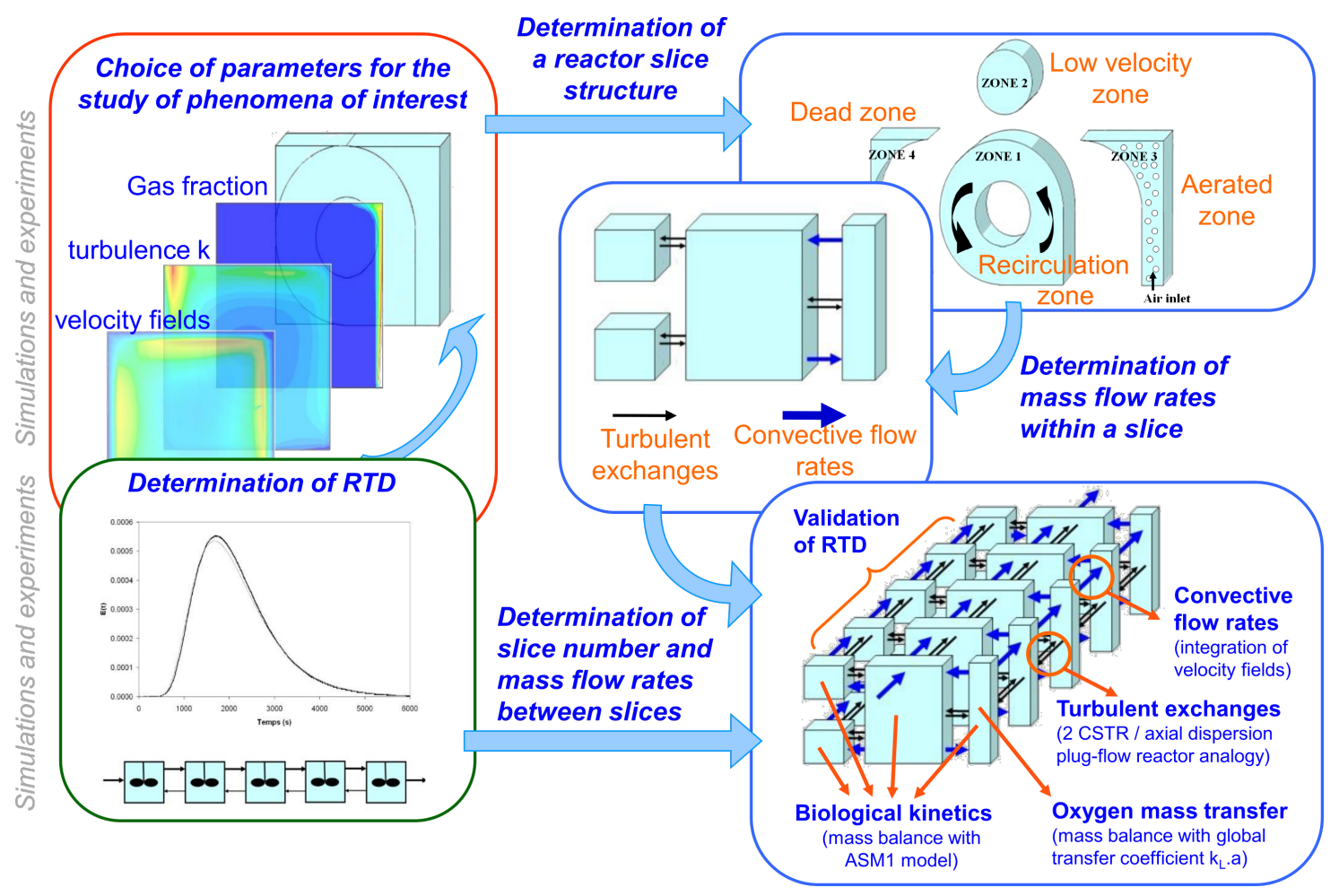

Figure 3: Construction procedure of Le Moullec (2010) compartmental model [58]

The Alvarado et al. [5] approach is close to the Le Moullec approach: it is based on hydrodynamics simulations and numerical RTD. Moreover, the two methods are designed to model water pond reactors. The construction method can be divided into four steps: division of the system in zones, calculation of the zones volumes, division of the zones into compartments and finally calculation of the exchanges fluxes. The system division is based on the analysis of velocity fields obtained by CFD simulations results. The reactor is divided in three zones: the first zone is based on the dominant direction of the flow (inlet to outlet), the second zone is based on the opposite direction of the main direction (backflow) and the third zone corresponds to the low velocity recirculation loop in the centre of the reactor. The volume of each zone and its division into compartments are determined thanks to the analysis of RTD curves. The created compartments can be considered as Continuous Stirred Tank Reactors or plug flow reactors with axial dispersion depending on the nature of the RTD curves. Convective fluxes are calculated with the velocity vectors and turbulent fluxes are calculated with the turbulence characteristics of the flow.

The Laakkonen and coworkers approach was used by Alopaeus et al. [4], Seppala et al. [96] to model the turbulence parameters of mixing in stirred tanks. This approach was improved afterwards by Nauha and Alopaeus [74, 75], Nauha et al. [76] to model algal growth in bubble column reactors. Zhao et al. [116] formulated a compartmental model based on the Alopaeus and coworkers approach to study the gas-liquid precipitation of $\mathrm{CO}_{2}-$ $\mathrm{Ca}(\mathrm{OH})_{2}$ system in an multiphase stirred crystallizer. The ultimate objective of this model development is to control processes for $\mathrm{CO}_{2}$ recovery and valorisation. The model was built obtaining energy dissipation rate with CFD simulation. Different zones of the reactor were empirically identified and then divided in a chosen number of compartments. Different model refinements were tested to find the best compartment number/size ratio to ensure calculation convergence independence.

For the study of wastewater treatment plants again, Rehman [89], Rehman et al. [90, 91] developed and compared different modelling ways. In this study, a full reactive CFD-biokinetics model is created to simulate the reagents and products concentration profiles in the system. The results of the simulations provide the local concentration values and allowed the calculation of the Cumulative Species Distributions representative of the reactor homogeneity also referred to as "Rehman-Nopens curves". To complete the study of the WWTP reactor, a compart- 
mental approach is introduced. The general structure is determined by following the operational characteristics of the studied reactor: aerated or not, anoxic, aerobic or anaerobic. The compartments are determined using the Oxygen concentration fields from CFD-biokinetics simulations and the Cumulative Species Distributions fields are plotted to identify the different inhomogeneous zones that needed more refinement (steep Rehman-Nopens curves indicate homogeneous zones, less steep ones indicate higher degree of heterogeneity). These construction steps are used for longitudinal and axial compartmentalisation. Finally, the convective and exchange fluxes between the compartments are calculated using CFD simulation results.

Tajsoleiman et al. [99] developed an automatic method for compartmental model creation. They created a zoning algorithm based on the sensibility of a defined "target variable" similar to a compartment definition criterion. Threshold values are set and the algorithm creates a zone mapping of the considered system by aggregating elementary cells from preliminary CFD studies. In the paper, the method is tested with a $700 \mathrm{~L}$ bioreactor with an impeller. The exchanged fluxes are calculated from CFD solving mass balance equations between the different compartments.

\subsection{Compartmental modelling of complex systems: hydrodynamics influenced by fast reactions}

One of the most important conditions in the classical way of compartmental models construction is the independence of the fluid dynamics from the phenomena of interest. However, complex systems can be modelled by compartmental approaches even if the fluid dynamics behaviour of the system strongly depends on the kinetics, but this need to be treated carefully. In most of the cases, the compartmental approach is built using CFD simulations including a simplified kinetic model.

Due to the specificity of combustion systems, Fichet et al. [31] developed a different kind of compartmental approach to model the NOx production in gas turbines. The construction was based on the simulation of fluid dynamics coupled with a simplified kinetic model. Two criteria were used to define the compartments: chemical criteria following the concentration of NOx production reagents and physical criteria depending on the flow pattern. Each created compartment is considered as a Continuous Stirred Tank Reactor so that complete 0D Kinetic Model can be solved independently in each compartment. This method is valid as long as the NOx formation reaction has no influence on the fluid dynamics behaviour: despite the combustion kinetics can strongly influence hydrodynamics, in this study, produced NOx (a few ppm) did not affect the hydrodynamics. For complex system wherein physics/chemistry/biology influence hydrodynamics, the choice of compartment construction criteria is crucial.

The compartmental approach of Du et al. [28] was developed to model the riser reactor of an FCC unit. First, the compartment network structure is determined based on the analysis of the flow fields directions generated by CFD simulations: 4 zones for the axial division and 3 zones for the radial division. Then, the compartment volumes and the exchange fluxes could be calculated. Subsequently, a numerical RTD simulation was run to determine the ideal reactor corresponding to each compartment. Finally, the FCC kinetic equations were added to the compartments. This work can be related with the model developed by Kaur et al. [45].

\subsection{Compartmental vs CFD and Systemic approach}

To assess the benefits of compartmental approaches in comparison with the historical modelling approaches, several developed compartmental approaches have been compared with both full systemic and full CFD modelling of the considered systems. The comparison to experimental data or other model results can be both useful to fit the compartmental model and validate it. Moreover, it can justify the choice of compartmental modelling approach over systemic and CFD modelling approaches.

The final compartmental model developed by Rigopoulos is validated comparing the dimensionless concentration obtained by CFD simulations to the compartment network for the studied bubble column reactor. Thanks to the fine-tuning between the compartmental model and the CFD simulations, the final difference of the concentration values is lower than $4 \%$ [93].

The compartmental model built in the study of Gresch et al. [34] was validated by modelling a wastewater treatment pilot reactor with ozonation process. RTD in the pilot reactor (Figure 4 left) was simulated using the compartmental model. The compartmental RTD curves were compared with RTD simulated with the full CFD model. The curves are shown in Figure 4 (right). The simulated RTD curves were also compared with experimental RTD 
curves obtained from measurements of a tracer concentration in the pilot reactor. The curves of compartmental RTD and experimental RTD are compared in Figure 4 (centre). It is possible to observe that the compartmental model curve fits with the experimental curve so it shows the validity of the compartmental approach to model reactor hydrodynamics. This comparison validates the use of compartmental modelling approach in this particular case of continuous wastewater treatment reactors with driving baffles.
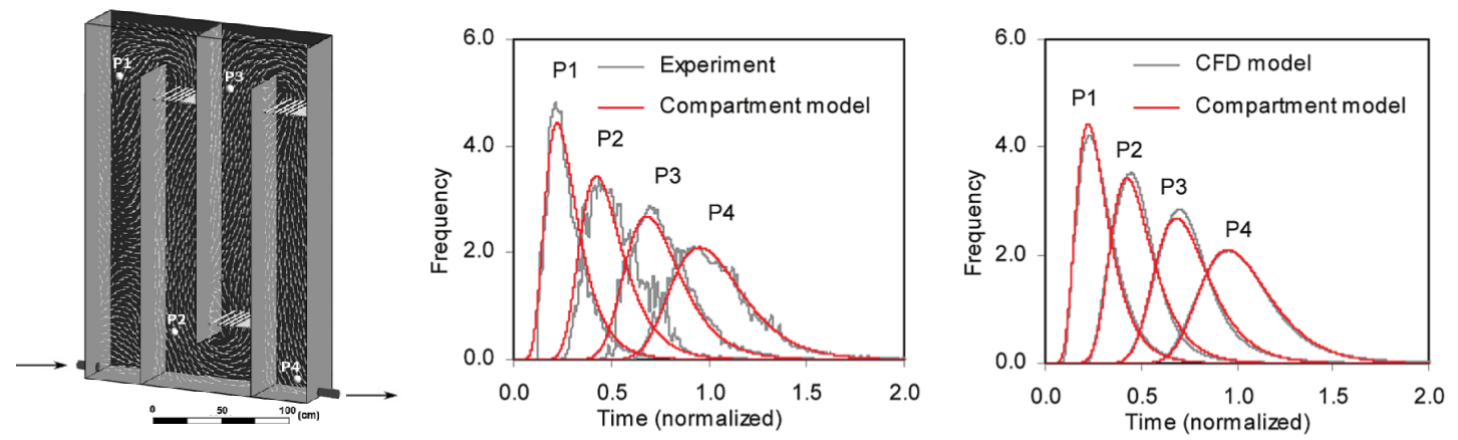

Figure 4: RTD curves at four sampling locations in the pilot reactor. These curves are normalised based on RTD curves at the outlet [34]

In the study of Le Moullec et al. [61], the compartmental model is compared both to a systemic model (ideal reactor network) and a full-CFD model [60]. The three approaches have an important influence on the way to model the hydrodynamic behaviour of the reactor but they are completed with the same coupled biokinetics model. The different approaches are used to model a wastewater treatment biological reactor. Le Moullec and coworkers conclude that they obtained the same information and the predictability as the CFD model with the compartmental model which is faster and simpler to develop. In a second study [62], the three different modelling approaches have been compared with experimental data and measurements (Figure 5). The concentrations of the different species involving in the biological reactions were plotted: oxygen, COD, nitrate and ammonium concentrations. The compartmental model gives the best predictions for oxygen concentration in all the experimental phases (Figure 5 left). The compartmental approach and the full CFD approach results are close for the prediction of COD and Nitrate concentrations. The three models are well representative of the COD concentration. CFD and Compartmental approaches has a tendency to under predict Nitrate concentrations up to $20 \%$ while the systemic approach have a tendency to over predict it up to $30 \%$. All the models failed to represent the ammonium concentration because of the sensitivity of the biokinetic model used in the different modelling approaches. In conclusion, the compartmental approach is a good solution to model the reactor hydrodynamics but it could be enhanced with a better parameter fitting in the kinetic model.
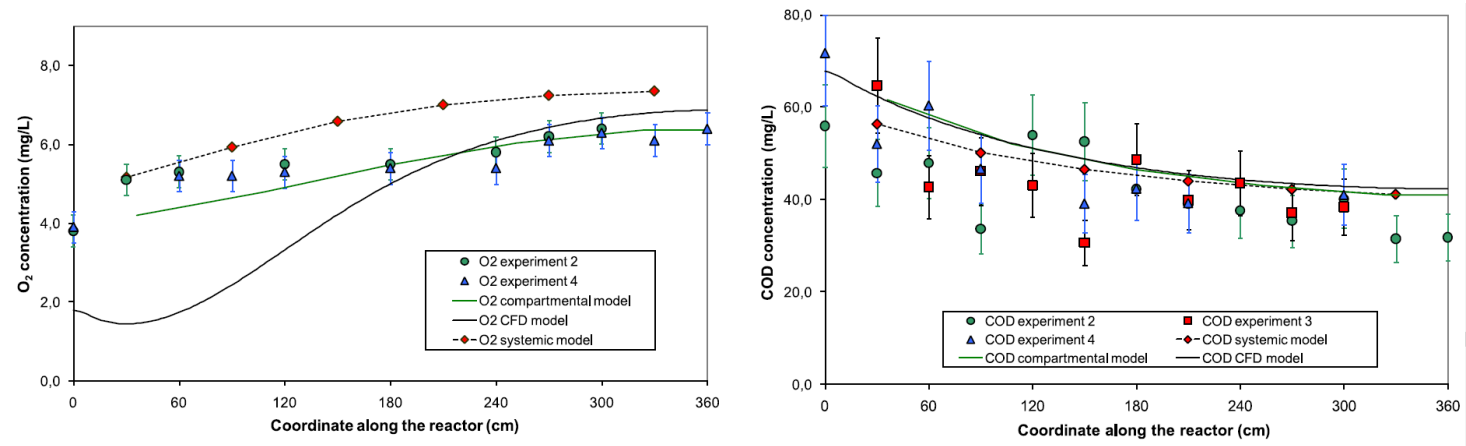

Figure 5: Comparison of Oxygen concentrations (left) and Chemical Oxygen Demand concentrations (right) for the three modelling approaches with experimental data [62]

In the Delafosse study, the developed compartmental approach is directly compared with experimental data 
from pilot scale reactor experiments [26]. The experimental data are also compared with both systemic and CFD approaches modelling results. The comparison is made by plotting mixing time and tracer concentration. The conclusion is the compartmental approach is quite easier to use and less time consuming than CFD with a better prediction of concentration fields and mixing time than systemic approach: the prediction of turbulent flows is too simplified with the systemic approach.

The compartmental approach built by Alvarado et al. [5] was developed in order to model a wastewater stabilisation pond. The results of the compartmental model were compared with a CFD simulation of the pond, three systemic models and experimental data from a full-scale pond. The systemic models consist of a network of CSTRs in series without recirculation or back-mixing flow, a network of CSTR with recirculation without back mixing flow and a CSTR network with recirculation and back mixing flow. The comparison was made by plotting the RTD curves obtained with the different models (Figure 6) and calculating the relative error between the hydraulic models and the experimental data. The different Tank In Series models were unable to model the RTD whereas the CFD approach and the compartmental model can predict the RTD curves: the Sum of Squares Error are respectively 2.5 and 2.8 in comparison with more than 4 concerning the systemic models. The conclusion of the study is that the compartmental model can predict the hydrodynamics accurately at low computational cost which is the easiest way to include some additional models such as biokinetic models.
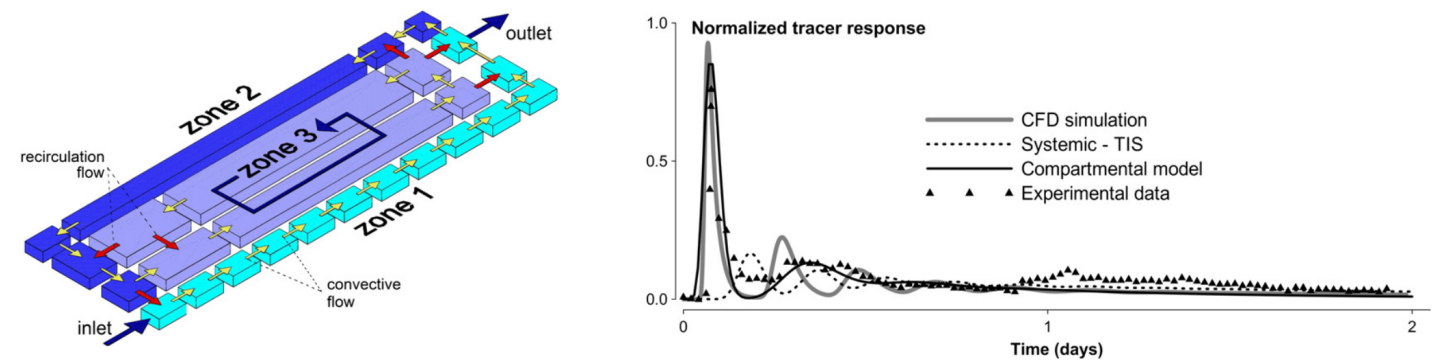

Figure 6: Representation of the compartmental model in the [5] study (left) and comparison of the RTD prediction with different models (right)

In the study of Du et al. [28], the constructed compartmental model called Equivalent Reactor Network model (ERN) was challenged with a simple plug flow model and a full-CFD model. The product distribution of the cracking reaction was modelled inside the FCC riser reactor with the three modelling approaches. The model results were compared with experimental data from a previous study. The CFD model and the ERN model predicts the mass fraction of the products well: the averaged relative error are respectively $3.2 \%$ and $4.79 \%$ and the values for each component stay below $6.09 \%$ and $6.24 \%$ respectively. In contrast, the average relative error for the piston model is $12.69 \%$ and the relative error reaches $20.73 \%$ for the gasoline mass fraction prediction. The ERN model prediction is better than the plug flow model prediction and it is pretty close to the CFD prediction with a significantly shorter computational time (more than 6 days for the CFD model and 5 seconds for the ERN model).

\section{Compartmental model limitations}

Compartmental modelling approach is suitable for the study of many complex systems with several objectives (system modelling, process control, scale-up...). However, the construction of compartmental models requires a complete study of the considered system. A large amount of information is needed to build an optimal model. Consequently, compartmental model construction of complex systems could require a strong knowledge of the related methodology.

As mentioned in the previous sections, compartmental modelling approaches are very often used when the phenomena of interest have no influence on the hydrodynamic behaviour of the system. If it is not the case, the choice of the definition criterion has to be carefully chosen, as illustrated in section 3.4.

Moreover, as demonstrated before, some applications of compartmental models such as scale-up are possible but sometimes, the compartment structure needs to be adapted (for example for dispersed bubble flows). 


\subsection{Synthesis of the review}

Through the review performed in the previous parts, the compartmental approaches have been inventoried. The distinction between the different approaches has been made following the evolution of the compartment definition (empirical, semi-automatic, fully automatic) and the fluid dynamics dependence on the phenomena of interest. This discrimination often follows increased model complexity as well as the chronological evolution of the compartmental models development. Some approaches result from the improvement of previous studies and express the logical evolution of models [26, 84], some are the adaptation of previous studies to a different application [75], some respond to a need in some particular application [28, 31].

Nevertheless, it is quite impossible to directly compare the different compartmental approaches because of the diversity of industrial applications and modelling objectives (Table 1). The next part analyses the different procedures for compartmental models construction, their validation and justifications compared to the existing approaches and the perspectives for the future of compartmental models development.

\section{Analyses}

In reviewed studies, the developed compartmental approaches were validated in different ways and compared with other modelling approaches. Furthermore, all the compartmental approaches have their own specificity particularly concerning the compartment construction criteria. Even though, it could be possible to propose a unified definition for compartmental modelling in Chemical Engineering by comparing the compartmental approaches with other modelling approaches and analyzing the compartmental approaches with one another.

\subsection{Model construction: construction criteria and fluxes calculation}

Each compartmental model relies on a common general pattern, but has its own construction schemes concerning the definition criteria. The compartment construction methods for each compartmental approach are detailed in Table 2.

Most compartmental approaches use the velocity fields as the main criterion for the compartments definition. As a consequence, validated CFD models are the cornerstone of a modern, compartmental modelling strategy in order to have the best hydrodynamic representativeness. Compartments are build around homogeneous velocity zone and an abrupt change in the gradient of the velocity field is the primary basis for determining the boundary between compartments.

However, the velocity fields study is often coupled with another parameter for the compartment definition. This second parameter must represent the phenomena of interest in the study. For example, with two-phase flow gas-liquid systems, the compartment definition is based on the dispersed phase fraction [28, 38, 61, 108]; for crystallization compartmental models, the construction parameter is based on the crystal size distribution (directly or indirectly linked with shear-stress that impacts coalescence and breakage) [41,42]. The study of characteristic times can be an important preliminary work to determine the most pertinent criteria for the compartment definition. For example, Guha et al. [35] compared the hydrodynamic time scale with the chemical phenomena time scale through the comparison between residence time and reaction time to justify the independence of the studied chemical phenomena from the hydrodynamic behaviour. The compartments were built small enough to ensure the residence time remains shorter than the chemical reaction time. For reactive systems, the concentration of the species can be chosen as compartments definition criteria [31, 90]. The criterion must be the concentration of the reagent or the product of the fastest reaction influencing the chemical reaction of interest [91, 92]. The choice of concentration as the definition parameter can be a problem in some complex cases because the initial CFD simulation must include a complete or a simplified kinetic model.

Complexity of the geometry meshing and CFD models are different according to the various approaches. CFD simulations can be run on unsteady or steady state regime depending on the wanted complexity. The flow can be simulated with all the coupled physico-chemical phenomena $[28,50,90]$, with simplified kinetic model $[25,31,34,84]$ or only with the fluid mechanic equations without any coupled phenomenon $[3,10,13,35,61]$. The choice of the complexity level of the CFD is driven by the system characteristics, by the influence of the phenomena on the hydrodynamics and foremost by the goal of the study. If the independence between kinetics and hydrodynamics is demonstrated, the hydrodynamic CFD simulations can be run without coupling models; if the 


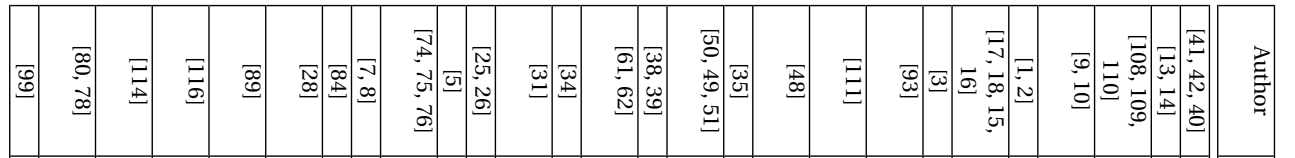

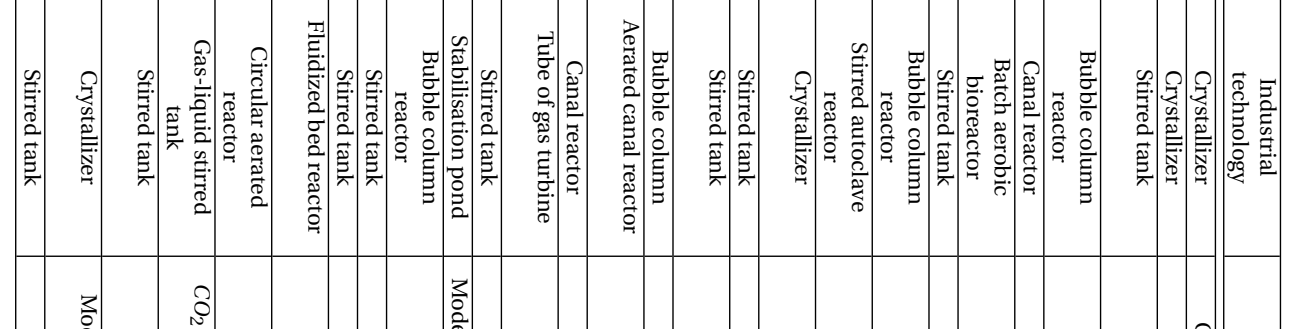

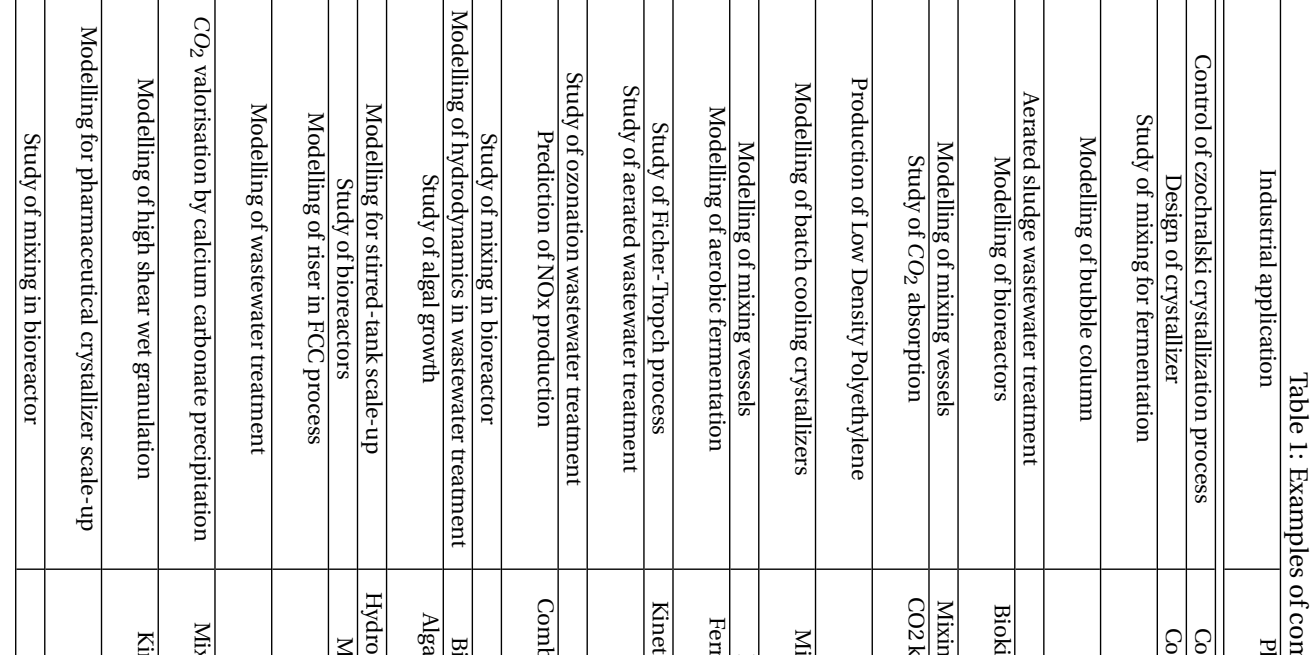

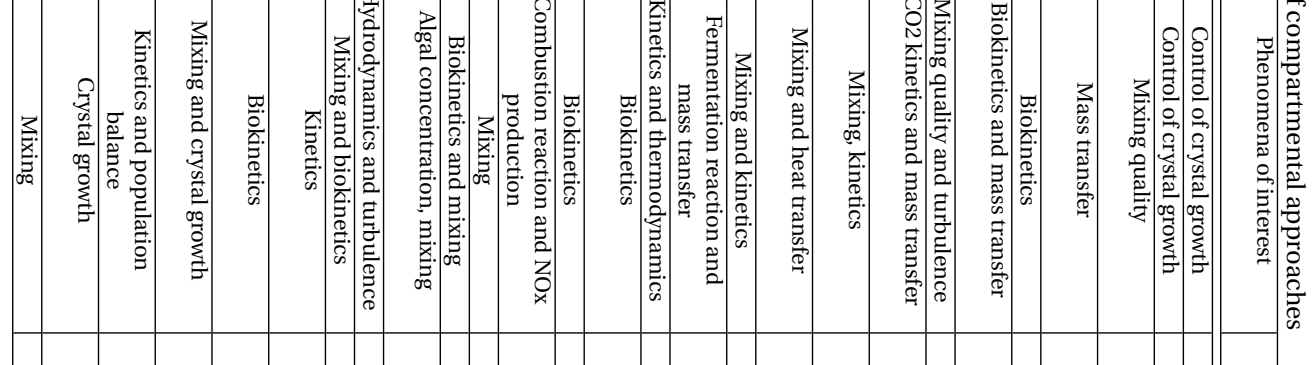

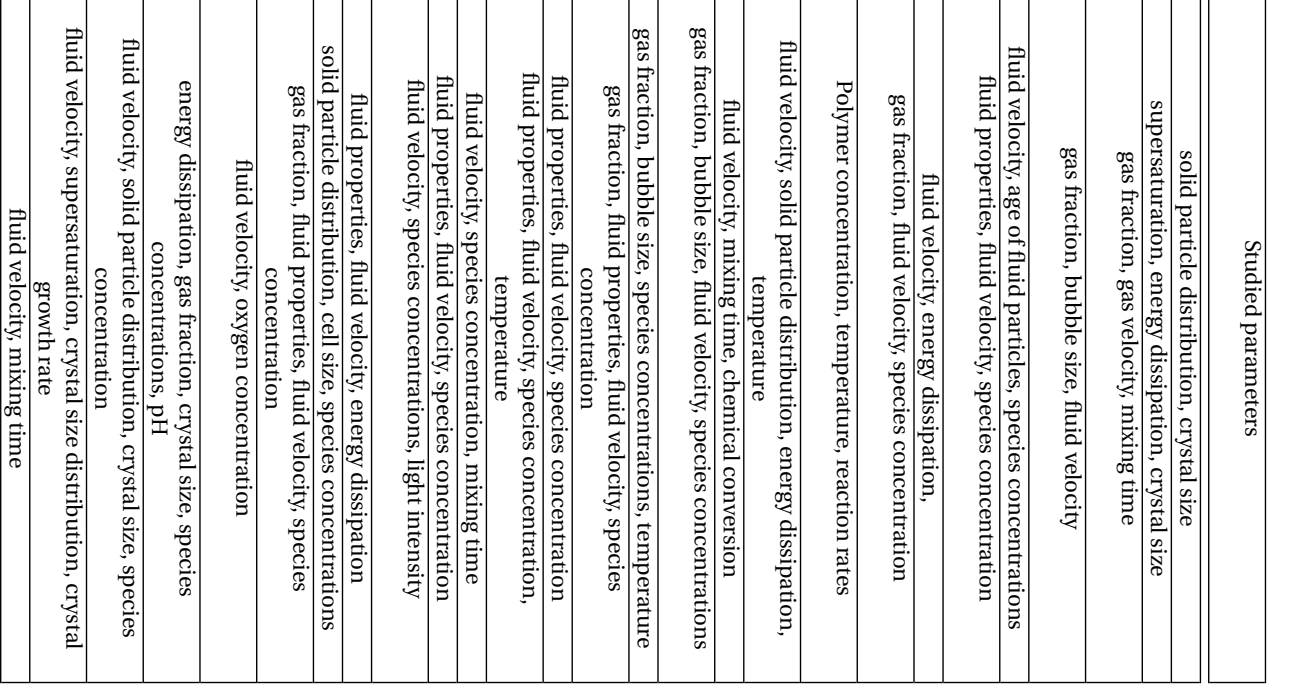


studied phenomenon has an influence on hydrodynamic parameters, this phenomenon must be taken into account for the hydrodynamic characterisation of the model. The preliminary CFD simulations can also be run in steady or unsteady state depending on the purpose of the model (investigation on the transient mode or on the common operation state) and the system behaviour over time (important variations that need process control). If too heavy, one can also run multiple steady states and then interpolate between compartments' volumes and exchange rates (De Mulder et al. [23]), building a so-called surrogate model. Anyway, the complexity of the model needed to define the compartmental model should be properly assessed, in the end the purpose of compartment modelling is to produce accurate model of complex system with less computational requirement but with good representation of important phenomena linked to the model objectives. Some systems with too close coupling between phenomena of interest and hydrodynamics could be difficult to model with today's compartmental approaches. By the way, the compartmental models of these complex sytems have to rely on strong CFD coupled simulations and the gain of compartmental modelling is potentially smaller. The choice of the modelling way could depend on this.

The approaches can be divided into two groups based on their compartment definition methods: an empirical definition or a mathematical definition. For the empirical definition, the number of compartments is chosen by observing the system geometry and the CFD results. The construction parameters are simulated and the compartments are visually defined by studying the obtained state variables fields. The refinement of the model is chosen beforehand. The mathematical definition is also based on the CFD results and the post processing is done with an algorithm that divides the system volume following given conditions on the construction criteria. For a given construction criterion, the mathematical conditions can be the homogeneity of the value within a compartment (e.g. concentrations, turbulence dissipation...), a negligible internal gradient, constant flow direction along a compartment frontier or a comparison between characteristic times. The refinement of the model is chosen by setting the cut values in the construction scheme or algorithm.

The defined construction criterion and the compartment construction method (empirical or mathematical) allow the determination of the compartments frontiers and volume. The gathering of the local information corresponding to CFD mesh cell values to obtain unified information within a compartment can be done by two different methods [36]: the method of successive volume division or the method of CFD cells aggregation $[15,16,25,28]$. The first one is the most used in the compartment approaches, the division can be empirical or numerical. The second one consists in identifying the cells corresponding to the centres of the future compartments and aggregate all the adjacent cells as long as the compartments construction conditions are checked. Given the important number of CFD cells, the aggregating cells method is always automated with a construction algorithm; various cell-aggregating algorithms are used and compared in Delafosse et al. [25].

In most of the cases, the compartments are considered as Continuous Stirred-Tank Reactor and their hydrodynamics behaviour is perfectly mixed. Only the approaches developed by Alvarado et al. [5] and Du et al. [28] model each compartment as a cascade reactor network based on the construction criterion. Also, compartments could be considered as plug flow reactors for example if there are some by-pass flows in the system.

To complete the compartmental model construction, the fluxes between the compartments are calculated. The exchange fluxes can be divided into two fluxes with different nature: the convective fluxes that represent the mean fluid flow circulation and the turbulent fluxes generated by the turbulent structures within the fluid [36]. The calculation of the convective fluxes is essential in all the compartmental models and it is done by integrating the flow field from CFD simulations results on the surface between two adjacent compartments. The calculation of the turbulent fluxes is much more complicated. Some compartmental approaches do not take the turbulent flux generations between the compartments into account, they only consider the convective flow as the modelling of all the fluid exchanges between the compartments. Guha et al. [35] was the first to introduce the turbulent flow calculation by considering a turbulent diffusion coefficient calculated from the turbulence parameters simulated by CFD. The turbulent diffusion coefficient method was then used in numerous compartmental approaches [5, 7 , 34, 84]. Le Moullec et al. [61] used another method to calculate turbulent flows, he considered an analogy between plug flow reactor with axial dispersion (Schmidt number calculation) andCSTR in series with back mixing flows [86]. 


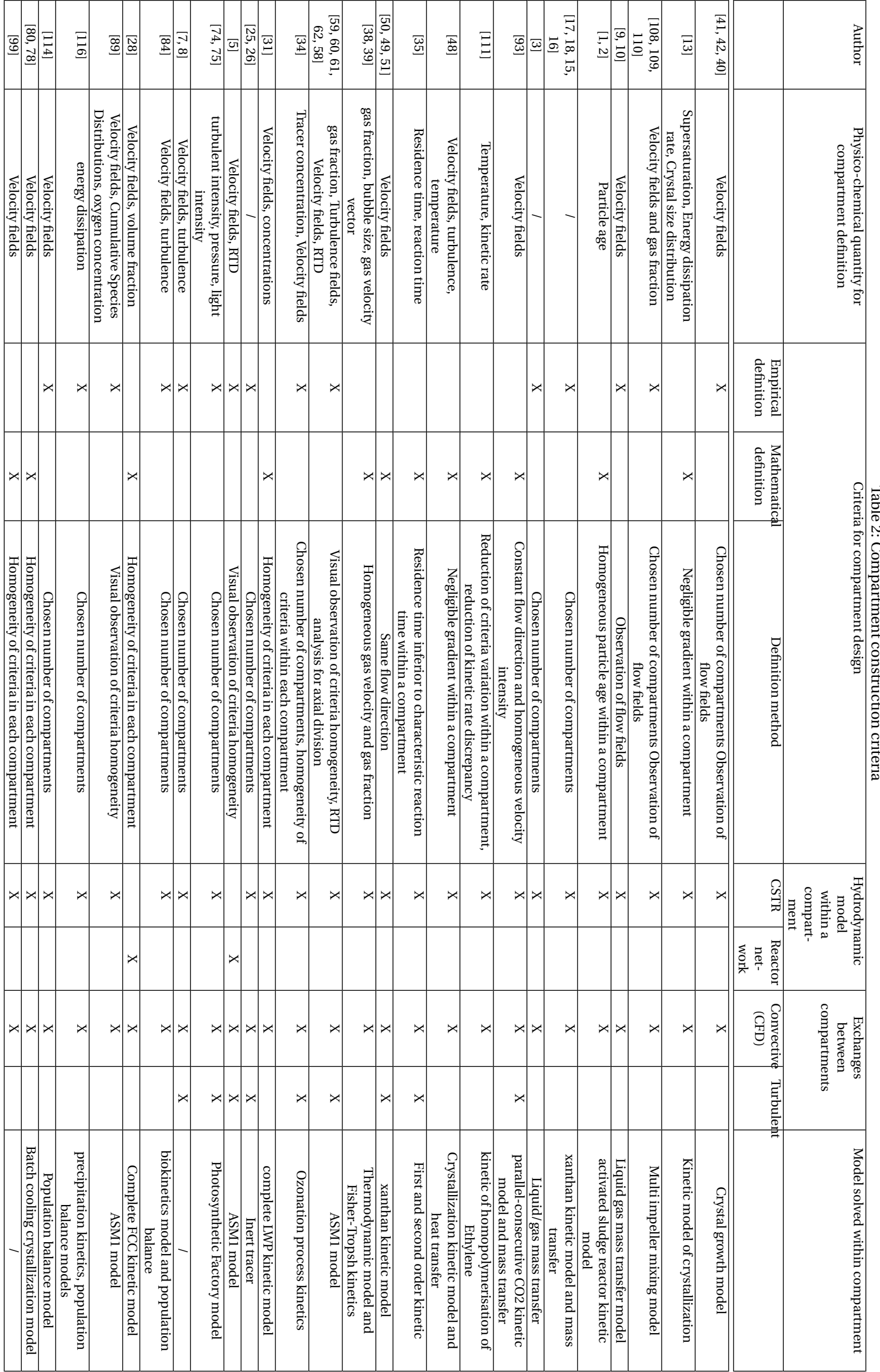




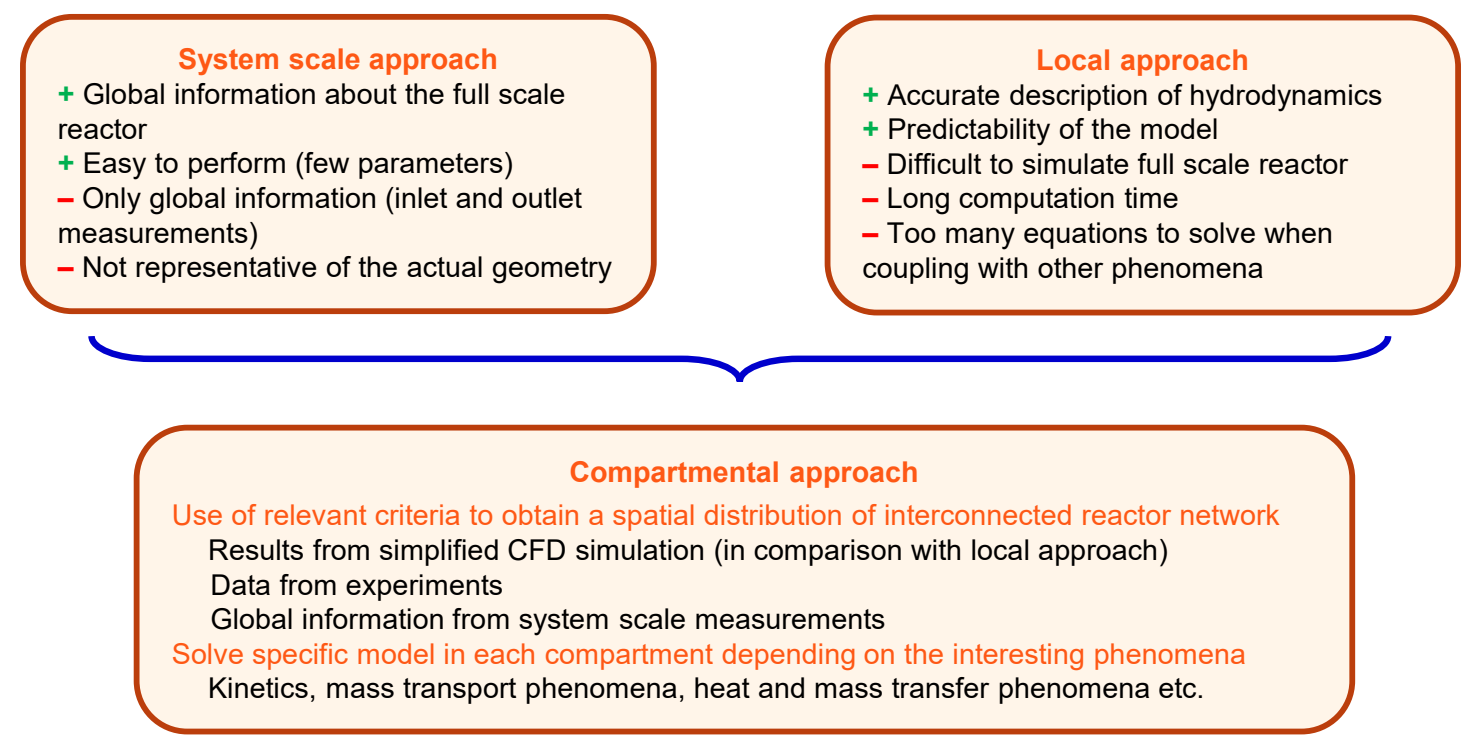

Figure 7: Benefits of compartmental approach

\subsection{Compartmental Models validation}

All the developed models must be validated in order to be effective. As demonstrated before, compartmental models development involve different modelling ways to construct the model structure. All the tools and methods used for compartmental model construction have to be validated too. In most of the cases, the system division into compartments is based on complete or simplified CFD simulations (following the construction criterion). The CFD simulations must be validated first for the compartmental model structure to be correct. The models validation needs complementary sets of data from other approaches (for example, experimental measurements can validate CFD simulations) or literature validated models from previous studies in similar cases. However, some elementary models are already verified and don't need further validation (e.g. well known velocity profiles in particular geometries); they can be used for compartmental models construction without intermediate validation.

In the most part of the cases shown in the review section, the compartmental models results are validated with proper experimental sets of data $[5,25,34,44,52,62,109,114,116]$. Sometimes, the results are compared with previous studies on similar systems $[74,75]$.

\subsection{Advantages of Compartmental Modelling}

Local measurements and simulations of fluid dynamics provide data for various parameters of the flow pattern (e.g. local velocities, viscosity, phase fractions, temperature, etc.). These local data characterize the system geometry, operating conditions and provide the actual mapping of the system. Local characterisation from CFD simulations or experiments are necessary to build the compartmental models structure. But experimental local approaches on industrial systems remains costly: to obtain a complete experimental characterisation, an important experimental setup is needed to get enough experimental values for a consistent mapping. Moreover, invasive probes can perturb the fluid dynamics behaviour of the system. In the same way, complete CFD simulations (hydrodynamics coupled with all the phenomena of interest and their dependencies) demand an important calculation time especially when all the coupling phenomena are simulated or when the system size becomes too important. In addition, local CFD simulations require a strong knowledge of the considered equations and models to obtain a meaningful result. In addition, compartmental models allow the implementation of more accurate physical models : for example, CFD multiphase simulations often assume incompressible gas phases whereas incompressible gas models can be patched in compartmental models.

That's why, in most cases, a simplified previous simulation of uncoupled fluid dynamics is done and the compartment network is designed analysing fluid property fields. Thus, the compartmental approaches gather infor- 

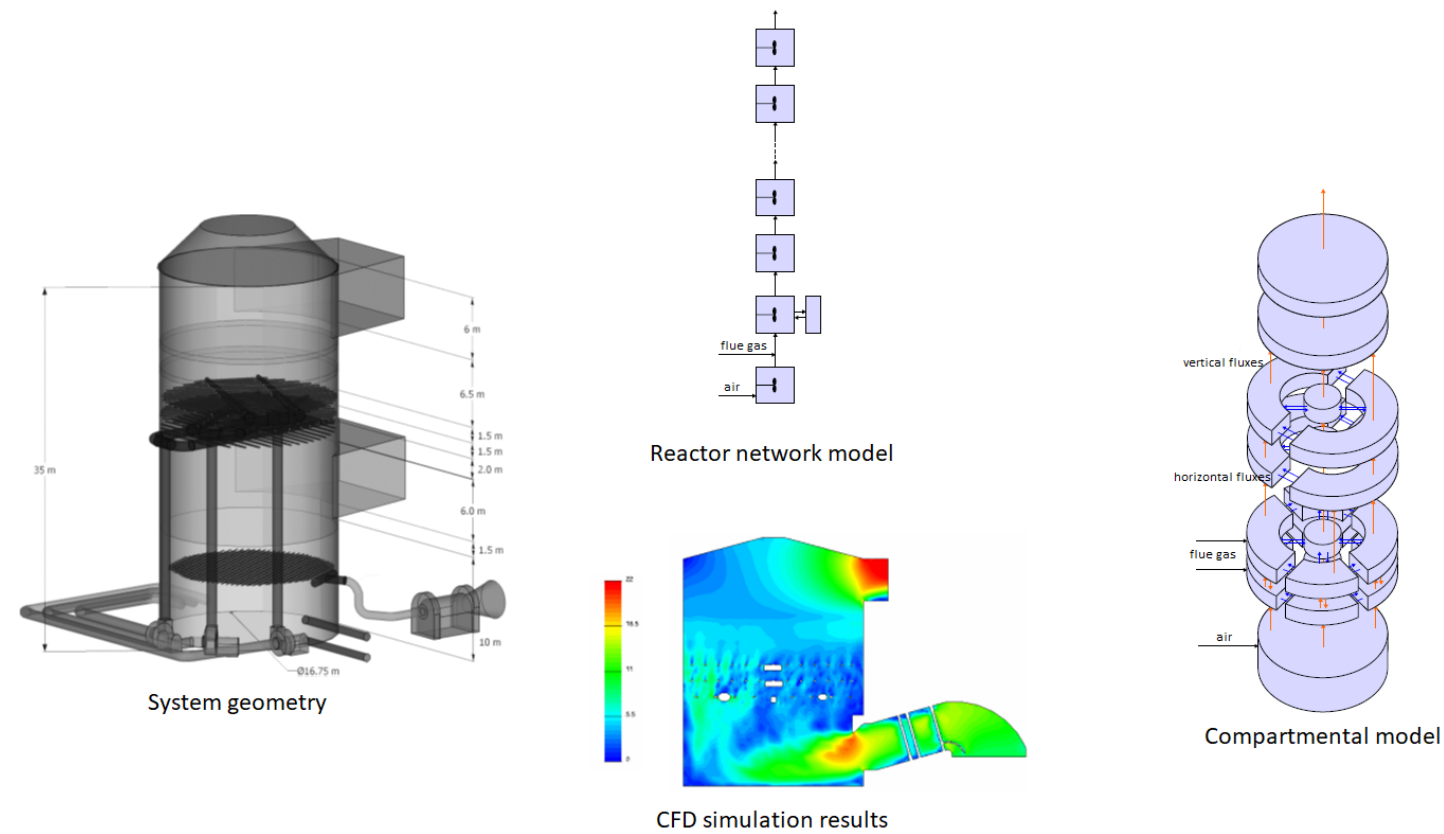

Figure 8: Examples of different modelling approaches applied to a Flue Gas Desulfurisation unit (adapted from [77])

mation from simulated fluid dynamics, mass transfer models and global balances (see Figure 7). Then, connections and exchanges between the compartments within the network are calculated with simulation results and experimental data. Finally, each compartment is a fully independent zone with its proper model depending on the phenomena of interests. The aggregation of systemic and local data provides a complete model of a multiphenomenon system with much shorter calculation time than a complete CFD simulation (Le Moullec et al. [61]). The compartmental approach is thus easier to implement for full-scale reactors (Alvarado et al. [5]) and the system model is fully predictable for example to reactor design (Bermingham et al. [13, 14]) or scale-up ([7, 80]). Compartmental models could be performed in operating plants as process control tools (Irizarry-Rivera and Seider $[41,42])$, flowsheet simulations or parameter fitting : the repetition of model calculation in these cases makes CFD simulations hardly operative

Compartment approaches allow the separation of phenomena modelling: the fluid dynamics behaviour of the system is simulated and a previous model is used to build the compartment structure and models of other phenomena are added thereupon. That is why compartmental approach simulation is much easier to perform than a coupled CFD simulation. The decoupling approach implies that phenomena of interest must not have influence on the system fluid dynamics. The more coupled the fluid dynamics are with studied phenomena, the more complex is the model construction; for example in fermentation processes, rheology is changing when biomass grows modifying the fluid hydrodynamic behaviour (Nunez-Ramirez et al. [79]). An overview of the different modelling approaches for a Flue Gas Desulfurisation unit is shown in Figure 8.

Compartmental models result to be good modelling approaches for many objectives (e.g. calibration, sensitivity analysis, scenario analysis, scale-up...) but for now, compartmental approaches development could remain insufficient to understand complex processes and perform reactor advanced design .

\subsection{Proposition of a unified definition of compartmental modelling}

Historically, names and definitions of both systemic and compartment model are strongly linked. At the beginning of systemic approaches in Chemical Engineering, Ideal Reactor Network models were often called Compartment Network Model [64] meanwhile Compartmental Models were often called network-of-zones models [44] or multiblock models [96]. Also, various works tried to simplify existing models dividing the studied system in several 
zones called compartments: for separation column processes, the dynamic low order models can be reduced by gathering several elements with the same behaviour into one "compartment" to reduce the number of differential equations $[12,47,83]$. The created "compartments" only have a functional coherence considering the initial model. These dynamic compartmental models are mostly similar to systemic models. In the pharmaceutical field, compartmental pharmacokinetic models can be used to model and predict drug transit and absorption inside the human body [52, 82, 113]. Again, the compartments used in these models are functional compartments considering the drug absorption model it is based on.

On the other hand, compartmental models are often named "reactors model" or "multi-zonal model". Nowadays, the distinction between Ideal Reactor Network models and Compartmental models is clear. In our case, the differences have been explained in the previous sections and that is why it seems important to propose a unified definition of what a compartmental model actually is.

\section{Unified definition of compartmental modelling}

Compartmental modelling approaches are often mistaken for systemic approaches due to their reactor network structure. But, the systemic reactor network construction is solely based on global information whereas the compartment network definition is based both on local and system scales information.

A compartmental model is a representation of a complex system based on the division of this system in functional compartments. It is based on a multi-scale description of the system fluid dynamics behaviour defining the system as a network of compartments (often CSTR, plug flow, reactor networks...). This compartment network is representative of the system geometry and spatial distributions of occurring phenomena: fluid dynamics, chemical reactions, biological reactions, transport phenomena, transfer phenomena, shear-induced physical processes (e.g. agglomeration, breakage and coalescence), etc. The compartment boundaries definition is based on the homogeneity of selected state variables set as construction criteria. The choice of these criteria depends on studied phenomena: homogeneity of reagent concentration to study (bio)chemical reactions, homogeneity for turbulence intensity to study mixing quality, the same flow rate direction to study transport phenomena, etc.

Compartment aggregate local information about fluid dynamics from CFD simulations and local measurements. They also use data from system scale calculation and system scale experimental characterisations. Convection and turbulent exchange fluxes between compartments are calculated for the complete network structures. Each compartment can be implemented with a complementary model according to their specificity within the studied system (e.g. ASM1 for water treatment, gas-liquid transfer and algal development model). For each compartment, a dedicated set of sub-model representing physical phenomena taking place in this compartment can be selected. This set of sub-model can be different from one compartment to the other.

\section{Construction methodology for compartmental models}

Based on the review and the analysis of the different compartmental approaches, it is possible to propose a general scheme of compartmental models construction (Figure 9).

This scheme can be divided in 6 main steps: the context of the study and the gathering of the system information, the preliminary study of the system, tools and methods available for the model construction, the compartment definition, the flux calculations and the model validation.

- Problem definition. The definition of the context of the study and the gathering of the system information are the first steps to identify the purpose of the model construction and the expected results. The modelling objectives influence the construction of the model: reactor design, process control, systems scale-up or better prediction for an existing process (increased model predictive power and reduced uncertainty for better decisions). Simultaneously, it is necessary to collect all the information concerning the system geometry and the process operation: local geometry, system parts and components, volume or occurring phenomena.

- Preliminary study. Then, the determination of the compartment construction criterion ensue from the first step. The determination takes into account phenomena of interest, the influence of the fluid dynamics on this phenomenon, the comparison of the different time scales and the available information about the system. The construction criterion can be merely fluid mechanic and transport phenomena related (e.g. velocity vectors, turbulence intensity, phase fraction...) or it can take into account hydrodynamics with coupled phenomena such as 


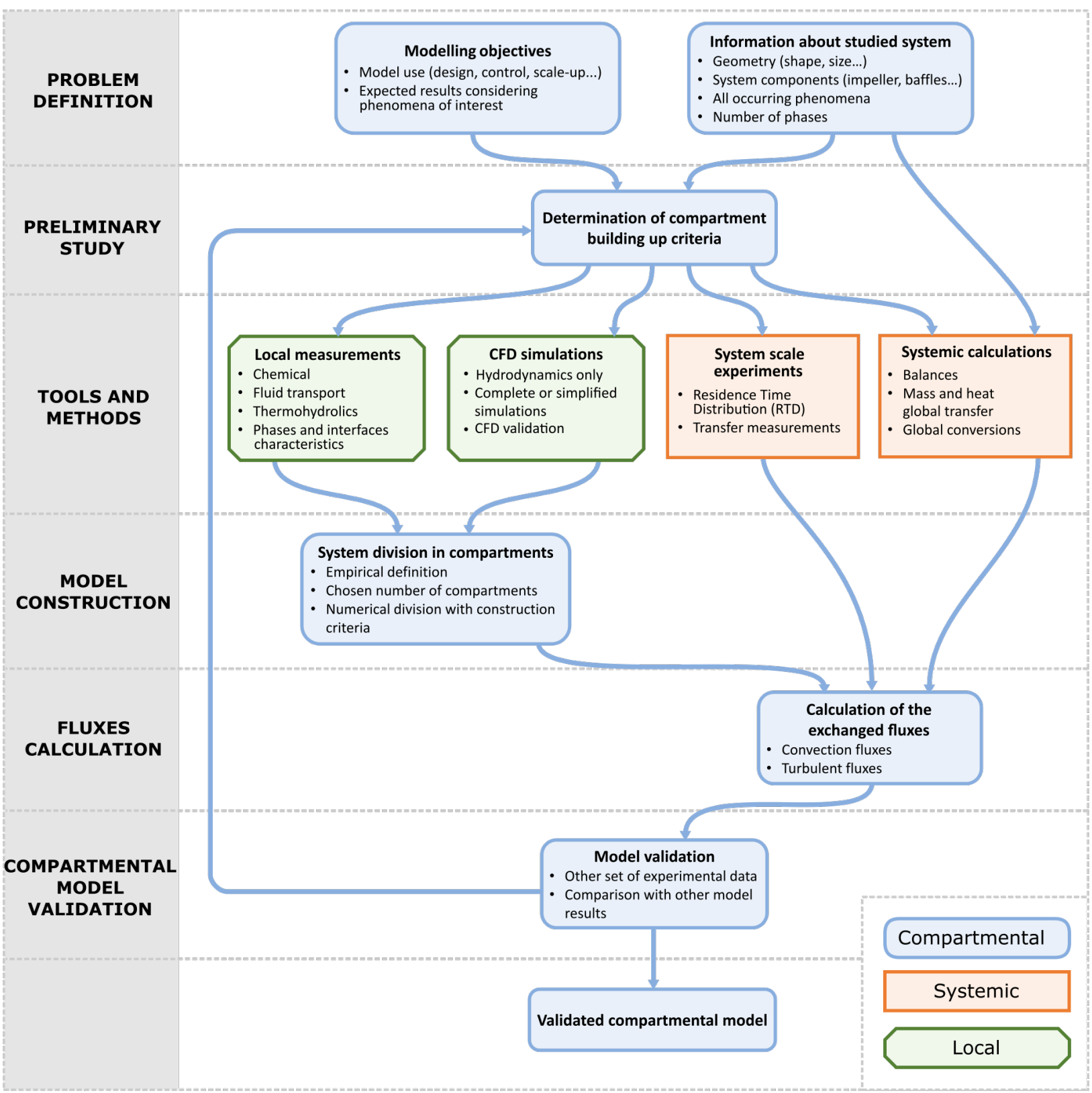

Figure 9: Proposed methodology for compartmental models construction

(bio)chemical reactions (e.g. reactant or product concentration, crystal size in crystallization processes, reaction time...).

- Tools and methods. Once the choice has been made, different studies of the system are carried to obtain the most complete characterisation of the system fluid dynamics. CFD simulations remain the most precise tool to get some local information (with reaction, most of the time without), but it can be complemented with experimental local experiments. The system scale information comes from the system balances calculation or experimental data (e.g. RTD tracer, global transfer measurements, etc.). More complex CFD simulations can be considered depending on the chosen compartment construction criterion. CFD simulations must be validated on their own to provide robust information to compartmental model construction. 
- Model construction and flux calculation. All the obtained local information is compiled and analysed to get an empirical definition of the compartments or an algorithm with given construction criterion values is used to divide the system into a compartment reactor network. The calculation of the exchanged fluxes is generally done by integrating the flow field from CFD simulations results on the surface between two adjacent compartments. The system scale data are used to complete the fluxes calculation and add system scale information to the model. This fluid dynamics model can be enhanced with all the other models to complete the system definition (e.g. heat or mass transfer model, kinetic model, bio-kinetic model).

- Compartmental model validation. The designed model could be validated with other data from previous study concerning the same kind of system, from another set of experimental data or from another set of full coupled CFD simulations results. If the validation tests fail, the compartment definition could be reconsidered, the correction can be minor concerning the refinement of the model or the cut value of the construction criterion. If the correction is more important, the determination criteria itself can be challenged.

\section{Critical reflections and future challenges}

Compartmental modelling approaches have, among others, emerged in order to tackle new problems and will continue to evolve, keeping its core principles as described in precedent section, to handle new challenges. This section highlights these main foreseen evolution pathways.

\subsection{Improvement in compartmental network generation}

\subsubsection{Automation of compartmental network generation}

As seen in the evolution of compartmental model in the literature, there is a clear trend to reduce the arbitrary during compartment network generation. Efforts has been put to define quantified compartment boundary selection criteria that could be used automatically or semi-automatically to define compartment network structure. Often these approaches generate models with very high number of compartments with very uneven volume distribution, this limiting the practicability of such approaches.

To solve this issue, compartmental network automation needs to come with mathematical techniques to adjust the compartmental network and/or exchange fluxes in order to produce efficient models that reach the main objectives of the study. To that extent, development of compartmental modelling could benefit from experience from other fields such as Process Intensification (PI) of Process Systems Engineering (PSE), where advanced optimisation techniques are employed to design a process - e.g. choose a set of operations (transformations of mass and energy), interconnected in a network (flowsheet) together with design and operating parameters (Preisig [88]). For instance, Baur et al. [11] used a generic non-equilibrium cell (functional model) that can be assembled to simulate a whole reactive distillation tray column. In process intensification, Demirel et al. [27] and Li et al. [67] used a generic block superstructure in which each block can implement various phenomena, leading to a panel of process configurations depending on the design problem and authorized connections between neighboring blocks. The automation criteria must take into account threshold values with a physical meaning according to the phenomena of interest. The number of compartments must be high enough to ensure the model precision but have to be balanced considering the calculation time of the model. A wise choice of compartment number ensures saving calculation time for example when a matrix-based resolution method is used.

As previously mentioned in section 3.3, Tajsoleiman et al. [99], proposed an automatic method for compartmental model construction. In the initialization step, criteria of model construction and the tolerance for each criterion are chosen. Then, the zoning algorithm aggregates cells from the CFD mesh according to the preset parameters. The compartments are determined by gathering the different zone maps established for the different criteria. Finally, the flows between compartments are identified and calculated.

The main difference with compartmental modelling is that networks and exchanged information are mainly based on functional objectives instead of spatial distribution, employed mathematical techniques could nevertheless be transposed for compartment modelling. Notwithstanding the potential hazards, the conceivable advantages are significant. As well as simulating new phenomena from first principles in a practical time-frame, a compartmental model can also be used as the precursor to a more advanced modelling framework. Knowledge 
of the system can be gained rapidly with a well-designed compartmental model, and this can feed forward into higher resolution modelling, safe in the knowledge that the extra investment in computational resources is likely to be worth it.

Another new theoretical approach, based on classical approaches in chemical engineering, can be also envisaged to help to automate compartmental model generation by calculation of local non-dimensional numbers. For example, the Reynolds number to locally quantify turbulence and mixing level, the Peclet number to quantify a number of compartments in series, the Hatta modulus to identify the type of compartments in polyphasic reactors (Hatta modulus gives information about the location of the reaction: in the liquid bulk, or at the interface, or both). The use of local non-dimensional numbers would be a keystone for the construction of self-adaptive compartmental models, described in the following section. An automatic calculation of such numbers would allow to determine the relative influence of phenomena and adapt the compartments structure with the fluctuation of operating conditions, such as hydrodynamics. For example, the Thiele Modulus characterizes the relative importance of catalytic reaction rate against diffusion rate. In compartmental models for porous catalyst, the Thiele Modulus calculation allows the identification of the catalyst regime (reaction regime or diffusion regime) and could influence the compartment construction automation.

\subsubsection{Self-adaptating dynamic compartmental network}

In compartment model construction, a key assumption regarding the compartment boundary selection is implicitly that it does not affect nor be affected by any physical or chemical disturbance (e.g. modification of system conditions, system geometry or fluid properties). Nevertheless, when modelling long-term dynamic effects that could induce a change in the flow field over time, the system could not always be accurately simulated by a static distribution of compartment. Compartment boundaries evolution are difficult to know conclusively in advance, as a consequence, more complex dynamic definition of boundaries could be considered, such as:

- Using variable compartmental boundary locations within a long-term simulation, based on information derived from several, discrete velocity fields generated by a series of steady-state CFD models. This approach needs a fully automatized compartment boundaries definition, that could be based on analysis of local non-dimensional numbers, as well as specific rule to decide to update the compartmental network.

- Using an adaptive flow rate between collateral compartments in a compartment network that envelop the highest number of compartment required: increasing the flow rate between them will increase the mixing rate. At a sufficient high flow rate, the two compartments will work as a single CSTR. This method was used by Potier et al. [86] with a model of cascade of CSTRs with back-mixing; its global hydrodynamics depends on the back-mixing flow rate between CSTRs.

Finally, for both discussed proposition and as stated in the section 5.1.1., both automating the compartmental boundary selection criteria and substantially increasing the number of compartments that would be acceptable in a compartmental model (e.g. into the hundreds), reduces the probability that a poorly chosen compartment selection process would affect the potential accuracy of any future model. This approach is a middle ground between a too simple network that gives poor representation and a too compartment heavy network that cripples the advantage of compartmental model over fully local approaches.

\subsection{New application fields for compartmental modelling}

Compartmental modelling is suitable to handle complex modelling scenarios while requiring low computing power. Thanks to this ability, compartmental modelling is a solution to model and simulate systems that have at least one these three characteristics: very large, high complexity, or if a rapid solution is mandatory while using limited computation power. Then, three kinds of new applications can be envisaged: study of new large systems, study of more complex phenomena and application with new objectives.

\subsubsection{Compartmental modelling for large systems}

The future of compartmental modelling approach lies in new phenomena studies and new applications for classical industrial processes. Moreover, compartmental approaches are strong multi-scale modelling tools so these approaches can be adapted to the largest industrial processes and huge natural milieus. Compartmental 
modelling with its low computing power is suitable to simulate very large processes and systems design by humans, such as power plants (e.g. cooling circuits Jourdan et al. [43]), constructed wetlands (Alvarado et al. [5]), hydroelectric dams, fish ladders, cities heat networks, air circulation in cities, etc.

By extension, compartmental modelling could be used to simulate natural milieus: rivers (e.g. self-purification processes, aeration [46]), lakes (e.g. lack of dissolve oxygen, lake turnover), oceans (e.g. evolution of carbon dioxide dissolution and distribution, thermohaline circulation), atmosphere (e.g. evolution of pollution, pollutant plums, atmospheric circulation), hyporheic zones (e.g. modelling of pollutant removal in biofilm), estuaries (e.g. nitrogen pollution, sediment), planets (e.g. simple models to study evolution over very long time), catchment basins, etc. For such systems, information from several methods are available, as in chemical engineering field. For instance, pollutant dispersion can be modelled using data from full-scale experiments, physical models of CFD (Lateb et al. [54]). The compartmental approach can therefore be well-suited to aggregate various experimental and numerical information without tedious computational effort. In the same way, compartmental model could be the perfect approach to model overall biochemical processes such as those happening in the body.

\subsubsection{Compartmental modelling for more complex systems}

This article already pointed out that compartmental approaches can be suitable for numerous phenomena. However, some complex phenomena have not yet been studied and compartmental modelling could be an effective modelling approach for these new, often tricky, cases. The following new applications could be considered as especially promising:

- A particular stake in compartmental modelling is the development of new approach allowing simulating processes where the transformation (reaction) has an influence on hydrodynamics: volume expansion, viscosity change, transient state, etc. The difficulty is the change in the structure of the compartment network. Envisaged solutions were presented in the previous section 5.1. Compartmental modelling could then be used for example in agro-food process, polymer production, polymerisation (injection moulding), combustion in transient state, safety studies (explosion), 3D printing, etc.

- Compartmental modelling can be also suitable for multi-scale phenomena simulations (Li et al. [65], Villermaux [104, 105], Sapre and Katzer [94]); the particular advantage being the possibility to group in only one compartment model (then one software) all the phenomena based not only on space distribution but also scale (Ge et al. [32], Dudukovic [29], Li et al. [66]). It appears a new approach: multi scale-compartmental modelling. It was mentioned by Bauer and Eigenberger [9, 10] as "multi scale zone model" but it remains a full perspective for achieved compartmental model. Most of chemical engineering processes could be concerned. For example, in wastewater treatment, reactions coupled with diffusion in flocs or biofilms could be simulated at the same time with other phenomena at the reactor scale.

At the root of compartmental modelling is the study of mixing in reaction vessel, introduction of multi-scale compartmental modelling could also open possible ways for better integration of macro- and micro-mixing in modelling and simulation. Also, the scope of phenomenon could be expanded with, for example, reactions at solid surfaces or interfaces that have, today, not been integrated in compartmental modelling. New development using these approaches would allow treating complex polyphasic and catalytic reactions; most probably in a multi-scale approach.

\subsubsection{New applications of compartmental modelling with new objectives}

Compartmental approaches have been used in numerous applications in Chemical Engineering. In the light of this experience, compartmental modelling range can be extended to more and more domains and objectives:

- Scale up/process design. There is compatibility between compartmental modelling and some new approaches of process design (Bashiri et al. [8], Oner et al. [80]). Then, using Compartmental modelling would favor fast exploration of different scenarios and help to rapidly make choice for process design. In the future, for given processes, we can envisage to propose pre-studied configurations with suitable compartmental modelling models; during the process design we should only to have to adjust some parameters.

- Data acquisition system design: not only for reactors conception but also for process monitoring design or data acquisition optimisation. For example, compartmental approaches can help to rapidly find suitable locations for sensors in complex inhomogeneous reactors. As it was first broached by Rehman et al. [90] to study the effect of oxygen sensors location in biological wastewater treatment plant reactors [23]. 
- Process control of large-scale systems, such as wastewater treatment plants, power plants, chemical plants, etc. Then the control process could use much better information than the ones used by PID control or from systems based on systemic model, and much faster than information from CFD that is impossible to directly use for control.

\section{Conclusion}

A thorough review of evolution of compartmental modelling approaches has been carried out. During the last thirty years, model development has shifted from observation based construction to computational fluid dynamic (CFD) calculation derived approaches and from empirical compartmental definition to systematic calculation of boundaries. Based on this review and associated observation a definition of compartmental models has been proposed:

A compartmental model is a representation of a system based on its division in functional zones called compartments. The compartmental model is representative of the system geometry and spatial distributions of occurring phenomena.

Compartmental model approach, in its modern development, can be seen as an advanced post processing step of CFD tools but is not a degraded CFD model. The key behaviour of flow and turbulence have the same representativeness with respect to the phenomena of interest. Today, compartmental model approach is mostly used to represent system in which the motion of fluid is not affected by other phenomena but new development begin to emerge to handle this retroaction in an efficient way.

Moreover, compared to new trends in complex system modelling, often centered on black box model build through statistical regression of large amount of data, compartmental model is a very interesting tool for knowledge development as the overall resulting model is fully explicit and impact of new condition can be studied in every compartment (i.e. location). It can also be more easily debugged, challenged and modified. Constituting sub-model can also be validated and then applied to other systems.

The key advantages of this approach are:

1. An effective compartment model could handle multiple, multiphysics phenomenological models (detailed kinetic reaction scheme, complex heat and mass transfer model, population balance, etc.) that could not be included in CFD analysis.

2. Observed deviation between fully detailed CFD model and compartment model show very small results deviation despite of very significant reduction in calculation time (3 orders of magnitude [61]) that open the door for simulating very large systems and perform real-time simulation.

3. Compared to more classic systemic models, a significantly better representation of local phenomena, mixing and turbulence for instance, allowing significantly more predictive results as well as a spatialisation of phenomena allowing new potential (sensor integration decision, geometry optimisation, etc.)

Based on the framework described in this work, numerous developments can be foreseen for the compartmental modelling approach. In the near future, it should be possible to offer a new solution to deliver high fidelity, easy to use, models for new systems integrating complex phenomena and/or need for real time simulation.

\section{Acknowledgments}

We would like to thank EDF and the ANRT association (Association Nationale de la Recherche et de la Technologie) for their support.

\section{References}

[1] J. Alex, R. Tschepetzki, U. Jumar, F. Obenaus, and K.-H. Rosenwinkel. Analysis and design of suitable model structures for activated sludge tanks with circulating flow. Water Science and Technology, 39(4):55-60, 1999.

[2] J. Alex, G. Kolisch, and K. Krause. Model structure identification for wastewater treatment simulation based on computational fluid dynamics. Water Science and Technology, 45(4-5):325-334, 2002. 
[3] A.H. Alexopoulos, D. Maggioris, and C. Kiparissides. Cfd analysis of turbulence non-homogeneity in mixing vessels: A two-compartment model. Chemical Engineering Science, 57(10):1735-1752, 2002.

[4] V. Alopaeus, P. Moilanen, and M. Laakkonen. Analysis of stirred tanks with two-zone models. AIChE Journal, 55(10):2545-2552, 2009.

[5] A. Alvarado, S. Vedantam, P. Goethals, and I. Nopens. A compartmental model to describe hydraulics in a full-scale waste stabilization pond. Water Research, 46(2):521-530, 2012.

[6] L. Angermann. Numerical simulations - Examples and applications in computational fluid dynamics. InTech, 2010. ISBN 978-953-307153-4.

[7] H. Bashiri, M. Heniche, F. Bertrand, and J. Chaouki. Compartmental modelling of turbulent fluid flow for the scale-up of stirred tanks. The Canadian Journal of Chemical Engineering, 92(6):1070-1081, 2014.

[8] H. Bashiri, F. Bertrand, and J. Chaouki. Development of a multiscale model for the design and scale-up of gas/liquid stirred tank reactors. Chemical Engineering Journal, 297:277-294, 2016.

[9] M. Bauer and G. Eigenberger. A concept for multi-scale modeling of bubble columns and loop reactors. Chemical Engineering Science, 54(21), 1999.

[10] M. Bauer and G. Eigenberger. Multiscale modeling of hydrodynamics, mass transfer and reaction in bubble column reactors. Chemical Engineering Science, 56(3):1067-1074, 2001.

[11] R. Baur, R. Taylor, and R. Krishna. Dynamic behaviour of reactive distillation tray columns described with a non-equilibrium cell model. Chemical Engineering Science, 56(4):1721 - 1729, 2001.

[12] A. Benallou, D.E. Seborg, and D.A. Mellichamp. Dynamic compartmental models for separation processes. AIChE Journal, 32(7):10671078, 1986.

[13] S.K. Bermingham, H.J.M. Kramer, and G.M. van Rosmalen. Towards on-scale crystalliser design using compartmental models. Computers \& Chemical Engineering, 22:355-362, 1998.

[14] S.K. Bermingham, H.J.M. Kramer, A.M. Neumann, and P.J.T. Verheijen. Measuring and modelling the classification and dissolution of fine crystals in a dtb crystalliser. Proceedings of the 14th International Symposium on Industrial Crystallisation, 1999.

[15] F. Bezzo and S. Macchietto. A general methodology for hybrid multizonal-cfd models: Part i. theoritical framework. Computers and Chemical Engineering, 28(4):501-511, 2004.

[16] F. Bezzo and S. Macchietto. A general methodology for hybrid multizonal-cfd models: Part ii. automatic zoning. Computers and Chemical Engineering, 28(4):513-525, 2004.

[17] F. Bezzo, S. Macchietto, and C.C. Pantelides. A general framework for the integration of computational fluid dynamics and process simulation. Computers \& Chemical Engineering, 24(2):653 - 658, 2000.

[18] F. Bezzo, S. Macchietto, and C.C. Pantelides. General hybrid multizonal-cfd approach for bioreactor modeling. AIChE Journal, 49(8): 2133-2148, 2003.

[19] Q. Chen and W. Xu. A zero-equation turbulence model for indoor airflow simulation. Energy and buildings, (28):137-144, 1998.

[20] Y.Q. Cui, R.G.J.M. Van Der Lans, H.J. Noorman, and K.Ch.A.M. Luyben. Compartment mixing model for stirred reactors with multiple impellers. Chemical Engineering Research and Design, 74(2):261-271, 1996.

[21] P.V. Danckwerts. Continuous flow systems. distribution of residence times. Chemical Engineering Science, 2(1):1-13, 1953.

[22] A. Dani, P. Guiraud, and A. Cockx. Local measurement of oxygen transfer around a single bubble by planar laser-induced fluorescence. Chemical Engineering Science, 62(24):7245 - 7252, 2007.

[23] C. De Mulder, U. Rehman, T. Flameling, S. Weijers, Y. Amerlinck, and I. Nopens. Compartmental modelling in a plant-wide context: Exploration and potential. WRRmod Seminar, 2018.

[24] J. de Rosnay. The Macroscope: A New World Scientific System. Harper Collins Publisher, 1979. ISBN 0-0601-1029-5.

[25] A. Delafosse, F. Delvigne, M-L. Collignon, M. Crine, P. Thonart, and D. Toye. Development of a compartment model based on cfd

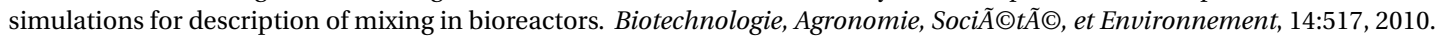

[26] A. Delafosse, M-L. Collignon, S. Calvo, F. Delvigne, M. Crine, P. Thonart, and D. Toye. Cfd-based compartment model for description of mixing in bioreactors. Chemical Engineering Science, 106:76-85, 2014.

[27] S.E. Demirel, J. Li, and M.M.F. Hasan. Systematic process intensification using building blocks. Computers and Chemical Engineering, 105:2-38, 2017.

[28] Y. Du, H. Zhao, A. Ma, and C. Yang. Equivalent reactor network model for the modeling of fluid catalytic cracking riser reactor. Industrial \& Engineering Chemistry Research, 54(35):8732-8742, 2015.

[29] M.P. Dudukovic. Reaction engineering: status and future challenges. Chemical Engineering Science, 65:3 - 11, 2010.

[30] T. Faravelli, L. Bua, A. Frassoldati, A. Antifora, L. Tognotti, and E. Ranzi. A new procedure for predicting nox emissions from furnaces. Computers and Chemical Engineering, 25(4):613 - 618, 2001.

[31] V. Fichet, M. Kanniche, P. Plion, and O. Gicquel. A reactor network model for predicting nox emissions in gas turbines. Fuel, 89(9): 2202-2210, 2010.

[32] W. Ge, F. Chen, J. Gao, S. Gao, J. Huang, X. Kiu, Y. Ren, Q. Sun, L. Wang, W. Wang, N. Yang, J. Zhang, H. Zhao, G. Zhou, and J. Li. Analytical multi-scale method for multi-phase complex systems in process engineering-bridging reductionism and holism. Chemical Engineering Science, 62:3346-3377, 2007.

[33] R.J. Goldstein. Fluid Mechanics Measurements. CRC Press, 1996. ISBN 978-156-032-306-8.

[34] M. Gresch, R. Brugger, A. Meyer, and W. Gujer. Compartmental models for continuous flow reactors derived from cfd simulations. Environmental Science \& Technology, 43(7):2381-2387, 2009.

[35] D. Guha, M.P. Dudukovic, P.A. Ramachandran, S. Mehta, and J. Alvare. Cfd-based compartmental modeling of single phase stirred-tank reactors. AIChE Journal, 52(5):1836-1846, 2006.

[36] J. Haag, C. Gentric, C. Lemaitre, and J.P. Leclerc. Modelling of chemical reactors: from systemic approach to compartmental modelling. International Journal of Chemical Reactor Engineering, 2018.

[37] S. Hocine, L. Pibouleau, C. Azzaro-Pantel, and S. Domenech. Modelling systems defined by rtd curves. Computers and Chemical Engineering, 32:3112-3120, 2008. 
[38] I. Iliuta, C.F. Petre, and F. Larachi. Hydrodynamic continuum model for two-phase flow structured-packing-containing columns. Chemical Engineering Science, 59(4):879 - 888, 2004.

[39] I. Iliuta, F. Larachi, J. Anfray, N. Dromard, and D. Schweich. Multicomponent multicompartment model for fischer-tropsch scbr. AIChE Journal, 53(8):2062-2083, 2007.

[40] R. Irizarry-Rivera. Fast compartmental monte carlo simulation of population balance models: Application to nanoparticle formation in nonhomogeneous conditions. Industrial \& Engineering Chemistry Research, 51(47):15484-15496, 2012.

[41] R. Irizarry-Rivera and W.D. Seider. Model-predictive control of the czochralski crystallization process. part i. conduction-dominated melt. Journal of Crystal Growth, 178(4), 1997.

[42] R. Irizarry-Rivera and W.D. Seider. Model-predictive control of the czochralski crystallization process. part ii. reduced-order convection model. Journal of Crystal Growth, 178(4):612 - 633, 1997.

[43] N. Jourdan, M. Kanniche, T. Neveux, and O. Potier. Compartmental modeling of particle settling in water basin. Proceedings of SFGP 2017 conference, pages 467-468, 2017.

[44] M. Kagoshima and R. Mann. Development of a network-of-zones fluid mixing model for an unbaffled stirred vessel used for precipitation. Chemical Engineering Science, 61:2852 - 2863, 2006.

[45] G. Kaur, M. Singh, J. Kumar, T. De Beer, and I. Nopens. Mathematical modelling and simulation of a spray fluidized bed granulator. Processes, 6:195-208, 2018.

[46] H. Khdhiri, O. Potier, and J.P. Leclerc. Aeration efficiency over stepped cascades: Better predictions from flow regimes. Water Research, 55:194-202, 2014

[47] S. Khowinij, M.A. Henson, P. Belanger, and L. Megan. Dynamic compartmental modeling of nitrogen purification columns. Separation and Purification Technology, 46:95-109, 2005.

[48] E. Kougoulos, A.G. Jones, and M. Wood-Kaczmar. Cfd modelling of mixing and heat transfer in batch cooling crystallizers. Chemical Engineering Research and Design, 83(1):30 - 39, 2005.

[49] M. Laakkonen, V. Alopaeus, and J. Aittamaa. Validation of bubble breakage, coalescence and mass transfer models for gas-liquid dispersion in agitated vessel. Chemical Engineering Science, 61(1):218-228, 2006.

[50] M. Laakkonen, P. Moilanen, V. Alopaeus, and J. Aittamaa. Dynamic modeling of local reaction conditions in an agitated aerobic fermenter. AIChE Journal, 52(5):1673-1689, 2006.

[51] M. Laakkonen, P. Moilanen, V. Alopaeus, and J. Aittamaa. Modelling local bubble size distributions in agitated vessels. Chemical Engineering Science, 62(3):721-740, 2007.

[52] J. Lainez-Aguirre, G. Blau, and L. Puigjaner. Building pharmacokinetic comaprtmental model using a superstructure approach. Computers and Chemical Engineering, 107:92-99, 2017.

[53] C. Laquerbe, J.C. Laborde, S. Soares, L. Ricciardi, P. Floquet, L. Pibouleau, and S. Domenech. Computer aided systhesis of rtd models to simulate the air flow distribution in ventilated rooms. Chemical Engineering Science, 56:5727-5738, 2001.

[54] M. Lateb, R.N. Meroney, M. Yataghene, H. Fellouah, F. Saleh, and M.C. Boufadel. On the use of numerical modelling for near-field pollutant dispersion in urban environments - a review. Environmental Pollution, 208:271 - 283, 2016.

[55] B.E. Launder, G.J. Reece, and W. Rodi. Progress in the development of a reynolds-stress turbulence closure. Journal of Fluid Mechanics, (68):537-566, 1975.

[56] D. Laurence, J.C. Uribe, and S.V. Utyuzhnikov. A robust formulation of the v2-f model. Flow, Turbulence and Combustion, (73):169-185, 2005.

[57] J. Laurent, P. Bois, M. Nuel, and A. Wanko. Systemic models of full-scale surface flow treatment wetlands: Determination by application of fluorescent tracers. Chemical Engineering Journal, 264:389 - 398, 2015.

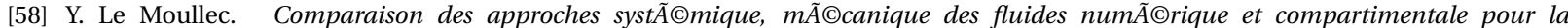

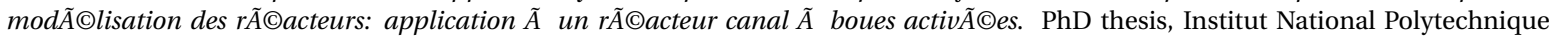
de Lorraine, 2008.

[59] Y. Le Moullec, O. Potier, C. Gentric, and J.P. Leclerc. Flow field and residence time distribution simulation of a cross-flow gas-liquid wastewater treatment reactor using cfd. Chemical Engineering Science, 63(9):2436-2449, 2008.

[60] Y. Le Moullec, O. Potier, C. Gentric, and J.P. Leclerc. Cfd simulation of the hydrodynamics and reactions in an activated sludge channel reactor of wastewater treatment. Chemical Engineering Science, 65(1):492-498, 2009.

[61] Y. Le Moullec, C. Gentric, O. Potier, and J.P. Leclerc. Comparison of systemic, compartmental and cfd modelling approaches: Application to the simulation of a biological reactor of wastewater treatment. Chemical Engineering Science, 65(1):343-350, 2010.

[62] Y. Le Moullec, O. Potier, C. Gentric, and J.P. Leclerc. Activated sludge pilot plant: Comparison between experimental and predicted concentration profiles using three different modelling approaches. Water Research, 45(10):3085-3097, 2011.

[63] E.R. Lee. Microdrop generation. CRC Press, 2002. ISBN 978-084-931-559-6.

[64] O. Levenspiel. Chemical Reaction Engineering. Wiley, 1998. ISBN 978-0-471-25424-9.

[65] J. Li, Y. Tung, and M. Kwauk. Multi-scale modeling and method of energy minimization in particle-fluid two-phase flow. In P. Basu and J.F. Large, , Circulating Fluidized Bed Technology II, Pergamon press, pages 89-103, 1988.

[66] J. Li, W. Ge, W. Wang, and N. Yang. Focusing on the meso-scales of multi-scale phenomena - in search for a new paradigm in chemical engineering. Particuology, 8:634-639, 2010.

[67] J. Li, S.E. Demirel, and M.M.F. Hasan. Process synthesis using block superstructure with automated flowsheet generation and optimisation. AIChe Journal, 64(8):3082 - 3100, 2018.

[68] D. Maggioris, A. Goulas, A.H. Alexopoulos, E.G. Chatzi, and C. Kiparissides. Use of cfd in prediction of particle size distribution in suspension polymer reactors. Computers \& Chemical Engineering, 22(Supplement 1):S315 - S322, 1998.

[69] D. Maggioris, A. Goulas, A.H. Alexopoulos, E.G. Chatzi, and C. Kiparissides. Prediction of particle size distribution in suspension polymerization reactors: effect of turbulence nonhomogeneity. Chemical Engineering Science, 55(20):4611 - 4627, 2000.

[70] B. Mayr, P. Horvat, E. Nagy, and A. Moser. Mixing-models applied to industrial batch bioreactors. Bioprocess Engineering, 9(1):1-12, 1993. 
[71] F.R. Menter. Two-equation eddy viscosity turbulence models for engineering applications. AIAA Journal, (32):1598-1605, 1994

[72] P. Moin and K. Mahesh. Direct numerical simulation: a tool in turbulence research. Annual Review of Fluid Mechanics, (30):539-578, 1998.

[73] E. Morin. On complexity. Hampton Press, 1990. ISBN 1-5727-3801-4.

[74] E.K. Nauha and V. Alopaeus. Modeling method for combining fluid dynamics and algal growth in a bubble column photobioreactor. Chemical Engineering Journal, 229:559-568, 2013.

[75] E.K. Nauha and V. Alopaeus. Modeling outdoors algal cultivation with compartmental approach. Chemical Engineering Journal, 259: 945-960, 2015.

[76] E.K. Nauha, Z. Kalal, J.M. Ali, and V. Alopaeus. Compartmental modeling of large stirred tank bioreactors with high gas volume fraction. Chemical Engineering Journal, 334:2319-2334, 2018.

[77] T Neveux and Y Le Moullec. Wet industrial flue gas desulfurization unit: Model development and validation on industrial data. Industrial \& Engineering Chemistry Research, 50(12):7579-7592, 2011.

[78] A. Norregaard, C. Bach, U. Kruhne, U. Borgbjerg, and V. Gernaey. Hypothesis-driven compartment model for stirred bioreactors utilizing computational fluid dynamics and multiple ph sensors. Chemical Engeneering Journal, 356:161 - 169, 2019.

[79] D.M. Nunez-Ramirez, L. Medina-Torres, J.J. Valencia-Lopez, F. Calderas, J. Lopez Miranda, H. Medrano-Roldan, and A. Solis-Soto. Study of the rheological properties of a fermentation broth of the fungus beauveria bassiana in a bioreactor under different hydrodynamic conditions. Journal of Microbiology and Biotechnology, 22(11):1494 - 1500, 2012.

[80] M. Oner, C. Bach, T. Tajsoleiman, G.S. Molla, M.F. Freitag, S.M. Stocks, J. Abildskov, U. Kruhne, and G. Sin. Scale-up modelling of a pharmaceutical crystallization process via compartmentalization approach. Computer Aided Chemical Engineering, 44:181 - 186, 2018.

[81] G.K. Patterson. Application of turbulence fundamentals to reactor modelling and scale-up. Chemical Engineering COmmunications, 8: 25-52, 1981.

[82] N. Pavarula and L.K. Achenie. A mechanistic approach for modeling oral drug delivery. Computers and Chemical Engineering, 57: 196-206, 2013.

[83] X. Peng, Z. Liu, J. Tan, and W. Bu. Compartmental modeling and solving of large-scale distillation columns under variable operating conditions. Separation and Purification Technology, 98:280-289, 2012.

[84] M. Pigou and J. Morchain. Investigating the interactions between physical and biological heterogeneities in bioreactors using compartment, population balance and metabolic models. Chemical Engineering Science, 126:267 - 282, 2015.

[85] H Pitsch. Large eddy simulation of turbulent combustion. Annual Review of Fluid Mechanics, (38):453-482, 2006.

[86] O. Potier, J.P. Leclerc, and M.N. Pons. Influence of geometrical and operational parameters on the axial dispersion in an aerated channel reactor. Water Research, 39(18):4454-4462, 2005.

[87] O. Potier, J. Brun, P. Le Masson, and B. Weil. How innovative design can contribute to chemical and process engineering development? opening a new innovaion path by applying the c-k method. Chemical Engineering Research and Design, (103):108-122, 2015.

[88] H.A. Preisig. Constructing and maintaining proper process model. Computers and Chemical Engineering, 34:1543 - 1555, 2010.

[89] U. Rehman. Next generation bioreactor models for wastewater treatment systems by means of detailed combined modelling of mixing and biokinetics. PhD thesis, Ghent University, Belgium, 2016.

[90] U. Rehman, M. Vesvikar, T. Maere, L. Guo, P. A. Vanrolleghem, and I. Nopens. Effect of sensor location on controller performance in a wastewater treatment plant. Water Science and Technology, 71(5):700-708, 2015.

[91] U. Rehman, W. Audenaert, Y. Amerlinck, T. Maere, M. Arnaldos, and I. Nopens. How well-mixed is well mixed? hydrodynamic-biokinetic model integration in an aerated tank of a full-scale water resource recovery facility. Water Science and Technology, 76(8):1950-1965, 2017.

[92] M. Reuss and M. Jenne. Compartmental model. conf. on Bioreactor Performance, Helsinger, Denmark, pages 63-75, 1993.

[93] S. Rigopoulos and A.G. Jones. A hybrid cfd-reaction engineering framework for multiphase reactor modelling: basic concept and application to bubble column reactors. Chemical Engineering Science, 58(14):3077-3089, 2003.

[94] A.V. Sapre and J.R. Katzer. Core of chemical reaction engineering: One industrial view. Industrial and Engineering Chemistry Research, 34(7):2202-2225, 1995.

[95] C.D. Scott-Pomerantz. The k-epsilon model in the theory of turbulence. PhD thesis, University of Pittsburgh, 2004.

[96] M. Seppala, M. Laakkonen, M. Manninen, V. Alopaeus, and J. Aittamaa. Development of automatic algorithm for combining cfd and multiblock modelling and application to flottation cell. 6th international conference on Computational Fluid Dynamics in the Oil and Gas, Metallurgical and Process industries, (Trondheim, Norway), 2008.

[97] M. Simcik, M.C. Ruzicka, A. Mota, and J.A. Teixeira. Smart rtd for multiphase flow systems. Chemical Engineering Research and Design, 90:1739-1749, 2012.

[98] D. Stropky, K. Pougatch, P. Nowak, M. Salcudean, P. Pagoria, I. Gartshore, and J. Yuan. Rtd (residence time distribution) predictions in large mechanically aerated lagoons. Water Science and Technology, 55(11):29-36, 2007.

[99] T. Tajsoleiman, R. Spann, C. Bash, K.V. Gernaey, J.K. Huusom, and U. Kruhne. A cfd based automatic method for compartmental model development. Computers and Chemical Engineering, 123:236-245, 2019.

[100] N. Takagaki and S. Komori. Air-water mass transfer mechanism due to the impingement of a single liquid drop on the air-water interface. International Journal of Multiphase Flow, 60:30-39, 2014.

[101] C. Tibirica, FJ. do Nascimento, and G. Ribatski. Film thickness measurement techniques applied to micro-scale two-phase flow systems. Experimental Thermal and Fluid Science, 34(4):463 - 473, 2010.

[102] H.K. Versteeg and W. Malalasekera. An introduction to Computational Fluid Dynamics - The Finite Volume Method (Second edition). Pearson, 2007. ISBN 978-013-127-498-3.

[103] J. Villermaux. Did you say process engineering? Chemical Engineering and Processing: Process Intensification, 18(3):123-127, 1984.

[104] J. Villermaux. Future challenges for basic research in chemical engineering. Chemical Engineering Science, 48(14):2525 - 2535, 1993

[105] J. Villermaux. Future challenges in chemical engineering research. Chemical Engineering Research and Design, 73:105-109, 1995.

[106] E. Vivaldo-Lima, P.E. Wood, A.E. Hamielec, and A. Penlidis. Calculation of the particle size distribution in suspension polymerization 
using a compartment-mixing model. The Canadian Journal of Chemical Engineering, 76(3):495-505, 1998.

[107] L. von Bertalanffy. General System Theory. Foundations, Development, Applications. George Braziller, New York, 1968. ISBN 0-80760453-4.

[108] P. Vrabel, R. Van der Lans, Y.Q. Cui, and K.. Luyben. Compartment model approach: Mixing in large scale aerated reactors with multiple impellers. Chemical Engineering Research and Design, 77(4):291-302, 1999.

[109] P. Vrabel, G. Rob van der Lans, C. Karel Luyben, L. Boon, and A. Nienow. Mixing in large-scale vessels stirred with multiple radial or radial and axial up-pumping impellers: modelling and measurements. Chemical Engineering Science, 55(23):5881 - 5896, 2000.

[110] P. Vrabel, G. van der Lans, F. van der Schot, C. Karel Luyben, B Xu, and S. Enfors. Cma: integration of fluid dynamics and microbial kinetics in modelling of large-scale fermentations. Chemical Engineering Journal, 84(3):463 - 474, 2001

[111] G.J. Wells and W.H. Ray. Methodology for modeling detailed imperfect mixing effects in complex reactors. AIChe Journal, 51(5):1508 $1520,2005$.

[112] V. Wizman, D. Laurence, M. Kanniche, P. Durbin, and A. Demuren. Modelling near-wall effects in second-moment closures by elliptic relaxation. International Journal of Heat and Fluid Flow, (17):255-266, 1996.

[113] L.X. Yu and G.L. Amidon. A compartmental absorption and transit model for estimating oral drug absorption. International Journal of Pharmaceutics, 186(2):119- 125, 1999.

[114] X. Yu, M.J. Hounslow, G.K. Reynolds, A. Rasmuson, I.N. Bjorn, and P.J. Abrahamsson. A compartmental cfd - pbm model of high shear wet granulation. AIChE Journal, 63(2):438-458, 2017.

[115] L. Zhang, Q. Pan, and G.L. Rempel. Residence time distribution in a multistage agitated contactor with newtonian fluids: Cfd prediction and experimental validation. Industrial \& Engineering Chemistry Research, 46(11):3538-3546, 2007.

[116] W. Zhao, A. Buffo, V. Alopaeus, B. Han, and M. Louhi-Kultanen. Application of the compartmental model to the gas liquid-precipitation of co2-ca(oh)2 aqueous system in a stirred tank. AIChE Journal, 63(1):378-386, 2017. 


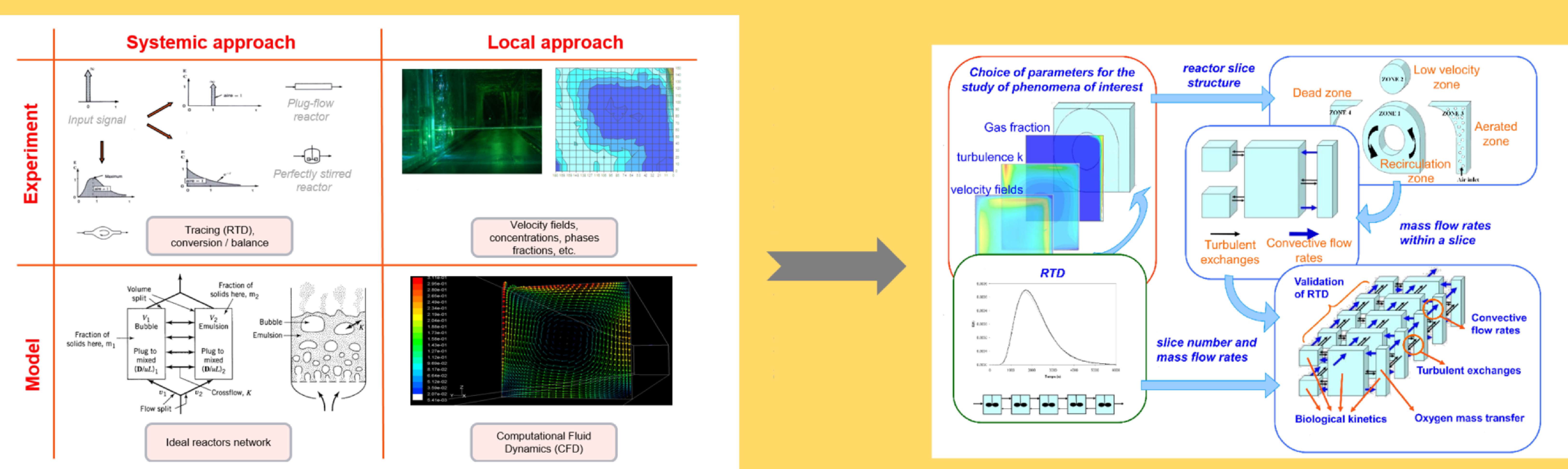

Compartmental modelling: a suitable approach to model and simulate complex systems 